\title{
Strategies of Flanker Coprocessing in Single and Dual Tasks
}

\author{
Ronald Hübner and Carola Lehle \\ Universität Konstanz
}

\begin{abstract}
In this study, the authors used a dual-task flanker paradigm to investigate the degree to which flankers are coprocessed with the target as a function of whether flankers have to be used as stimuli for a second task. A series of experiments, in which performance in dual tasks was compared with that in single tasks, revealed that participants had a strong tendency to coprocess flankers to a large degree in dual tasks, even if this impaired performance. Coprocessing of flankers was reduced only when totally irrelevant flankers were presented at the beginning of a trial or single tasks were performed on the great majority of trials within a block. The results suggest that it was demanding to process targets and flankers serially when both had to be used for a dual task. As a consequence, target and flankers were processed in parallel, even if this was nonoptimal for target selection.
\end{abstract}

Keywords: dual tasks, flanker task, parallel processing, attention, processing strategies

On the basis of learned stimulus-response (S-R) associations, stimuli compete in a bottom-up manner for their representation and for the control of action. When stimuli differ, there can be interference and response conflicts (e.g., Desimone \& Duncan, 1995). Consequently, for goal-directed behavior to be maintained, relevant stimuli have to be prioritized and irrelevant ones ignored. Details of the selection mechanisms involved in target prioritization have been investigated with so-called conflict paradigms, such as the Eriksen flanker task (B. A. Eriksen \& Eriksen, 1974), the compound-stimulus task (e.g., Briand, 1994; Hübner \& Volberg, 2005; Paquet \& Merikle, 1988), the S-R compatibility task (e.g., Kornblum, Hasbroucq, \& Osman, 1990; Marble \& Proctor, 2000; Proctor \& Vu, 2002), and the Stroop task (Stroop, 1935). Various results indicate that stimulus selectivity is limited in that some processing of irrelevant stimuli can hardly be avoided (e.g., C. W. Eriksen \& Hoffman, 1973; Paquet, 2001; Paquet \& Craig, 1997). Moreover, it has been proposed that this processing of irrelevant stimuli is largely independent of the strategies adopted by participants (e.g., Miller, 1991).

Whereas the effects and mechanisms of involuntary stimulus processing have been investigated intensively, relatively few studies have examined the voluntary processing of currently irrelevant but potentially informative stimuli. One of the few exceptions is the work of Logan and his colleagues (Logan \& Zbrodoff, 1979, 1982; Logan, Zbrodoff, \& Fostey, 1983; Logan, Zbrodoff, \& Williamson, 1984). Using Stroop-like tasks, Logan and colleagues have demonstrated that attentional strategies often modulate interference from irrelevant information, where a strategy was defined as an "optional organization of cognitive processes designed to achieve some goals in some task environment" (Logan et al., 1983,

Ronald Hübner and Carola Lehle, Kognitive Psychologie, Fachbereich Psychologie, Universität Konstanz, Konstanz, Germany.

We thank Marco Steinhauser for his valuable comments on an earlier version of this article.

Correspondence concerning this article should be addressed to Ronald Hübner, Fachbereich Psychologie, Universität Konstanz, Fach D29, D-78457, Konstanz, Germany. E-mail: ronald.huebner@uni-konstanz.de p. 485). Logan et al. (1983) investigated the construction and change of strategies by manipulating the degree to which the irrelevant dimension contained predictive information about the relevant dimension. Their results show that attention can strategically be divided between stimulus dimensions according to the validity of the irrelevant information. In general, Logan and colleagues have shown that the applied strategy depends on the task environment, on task demands, and on the available resources and abilities of the participant. Similar conclusions have also been drawn from results observed with S-R compatibility tasks (e.g., Marble \& Proctor, 2000; Proctor \& Vu, 2002; Proctor, Vu, \& Marble, 2003; Vu \& Proctor, 2004).

The present study was designed to examine the limits on strategies for information selection in a conflict paradigm. Previously, strategic aspects of selection have been investigated by varying the proportion of incongruent trials in the Stroop task (Logan \& Zbrodoff, 1979, 1982; Logan et al., 1983, 1984), in the S-R congruency task (Marble \& Proctor, 2000), and in the flanker task (e.g., Gratton, Coles, \& Donchin, 1992). Here, we went a step further and varied the relevance of flankers in dual-task flanker experiments. In the standard flanker task, a single task has to be performed on a target while simultaneously present flankers have to be ignored. In our study, however, a dual task was required on some trials (i.e., a task also had to be performed for the flankers). In a series of experiments, we asked the following questions: How do participants deal with momentarily irrelevant flankers if the flankers have to be used for a second task? Do they suppress flanker processing to protect the current task, or do they process the flankers? To what extent are participants able or willing to adapt their processing strategies to changing conditions? As our results show, participants have a strong tendency to coprocess flankers on dual-task trials to a larger degree than on single-task trials. However, before reporting our experiments in detail, we present a short overview of relevant paradigms.

\section{THE ERIKSEN FLANKER PARADIGM}

In the flanker task (B. A. Eriksen \& Eriksen, 1974), a target stimulus is usually presented in the center of the display, and a 
speeded classification response is required. Task-irrelevant flankers are presented to the left and to the right of the target. Because the same set of stimuli is usually used for the flankers as well as the target, a common S-R mapping applies to all items, and flankers can be mapped onto the same or the opposite response to the target (on congruent and incongruent trials, respectively). In some cases, there are also neutral flankers, which are not assigned to any response.

The degree of flanker coprocessing can be assessed by comparing the effect of the flankers on target processing across the different flanker conditions. For instance, performance when there are incongruent flankers is usually impaired relative to performance when the flankers are congruent-the flanker congruency effect (FCE; B. A. Eriksen \& Eriksen, 1974; C. W. Eriksen \& Schultz, 1979). The size of the FCE can be considered an indication of how intensively the flankers are coprocessed with the target.

Several mechanisms have been proposed to explain flanker effects, most relying on concepts of spatial selection. Space-based accounts assume that attention is distributed over a contiguous region of the visual field. Stimuli that fall within this "spotlight" of attention are preferentially processed, whereas stimuli outside this area are ignored (e.g., B. A. Eriksen \& Eriksen, 1974; Posner, Snyder, \& Davidson, 1980). A specific version of this idea is the zoom-lens model proposed by C. W. Eriksen and Schultz (1979). They proposed that after stimulus onset, attention is distributed over a wide area, so the target and flankers are processed in parallel. Because Eriksen and Schultz's model also assumes a continuous flow of information, it follows that partial information from both target and flankers activates responses early in the process. As the processing continues, though, spatial attention is increasingly focused on the target, improving performance. In later studies, these ideas were developed further in the dual-phase model (Coles, Gratton, Bashore, Eriksen, \& Donchin, 1985; Gratton et al., 1992; Gratton, Coles, Sirevaag, Eriksen, \& Donchin, 1988). According to the dual-phase account, the FCE is attributable to the concurrent activation of competing responses in the early phase of processing. However, it has also been hypothesized that the diameter of the attentional focus cannot be smaller than about $1^{\circ}$ of visual angle (B. A. Eriksen \& Eriksen, 1974; Paquet \& Craig, 1997; Yantis \& Johnston, 1990), which limits the selectivity of the focused phase as well.

Some studies have also investigated strategic modulations of the flanker effect. Gratton et al. (1992), for instance, presented cues indicating whether a congruent or an incongruent stimulus was likely to occur. Their data revealed that participants responded relatively quickly if the cue indicated a congruent trial but were rather slow if it signaled an incongruent one. From these results, Gratton et al. (1992) concluded that if participants expect congruent flankers, they will respond on the basis of the early, parallel phase of processing because the risk of an error is relatively low in this case. However, if incongruent flankers are expected, then the response will be based on the slow, but more reliable, focused phase.

Apart from this example, relatively few studies have been concerned with strategic influences on flanker effects. Moreover, in almost all studies, only single tasks have been considered, which rather limits manipulations of flanker coprocessing. Therefore, in the present study, we also included dual tasks.

\section{DUAL-TASK PROCESSING}

In experiments using the so-called psychological refractory period (PRP; Telford, 1931) paradigm, participants have to respond to a stimulus $\left(\mathrm{S}_{1}\right)$ according to a task $\left(\mathrm{T}_{1}\right)$. During the processing of $T_{1}$, a second stimulus $\left(\mathrm{S}_{2}\right)$ is presented with a certain stimulus onset asynchrony (SOA), for which a second task $\left(\mathrm{T}_{2}\right)$ is required. A typical result observed with this paradigm is the so-called PRP effect-that is, the result that the response time for $\mathrm{T}_{2}\left(\mathrm{RT}_{2}\right)$ increases with decreasing SOA. The PRP effect has been interpreted as evidence for a central (response-selection) bottleneck (e.g., Pashler, 1984). Whereas it is assumed that the precentral perceptual and the postcentral motoric stages of two tasks can proceed in parallel, it is supposed that response selection can take place for only one task at a time.

However, there are several alternative accounts of dual-task performance that do not make the bottleneck assumption (e.g., Logan \& Gordon, 2001; Meyer \& Kieras, 1997; Tombu \& Jolicœur, 2003). For instance, the central capacity sharing (CCS) model (McLeod, 1977; Navon \& Miller, 2002; Tombu \& Jolicœur, 2003) assumes that central capacity is limited but can be strategically divided between different tasks. Because it is possible to allocate all capacity to the first task, the CCS model contains the central bottleneck as a special case. Additionally, however, the CCS model can easily explain the fact that response time for $\mathrm{T}_{1}$ $\left(\mathrm{RT}_{1}\right)$ often decreases with an increasing SOA between $\mathrm{S}_{1}$ and $\mathrm{S}_{2}$ (e.g., Pashler, 1990, 1991; Smith, 1969; Tombu \& Jolicœur, 2002), which is incompatible with the idea of a central bottleneck.

In dual-task flanker experiments, results are even more complicated than they are in standard dual tasks, because the same S-R mapping applies for both tasks. In this case, one can expect not only unspecific effects of $\mathrm{S}_{2}$ processing on $\mathrm{RT}_{1}$ but specific ones as well (see also Hommel, 1998). For instance, Logan and Schulkind (2000) used numerals as stimuli for $T_{1}$ as well as $T_{2}$, with $S_{2}$ being presented close to $S_{1}$. Because the participants had to perform the same task (parity or magnitude judgments) with both stimuli, from the perspective of $\mathrm{T}_{1}$, the situation was similar to the flanker task. Accordingly, $\mathrm{S}_{2}$ produced a substantial FCE on $\mathrm{RT}_{1}$. To account for this and other effects, Logan and Gordon (2001) developed their executive control theory of visual attention (ECTVA), in which (similar to the CCS model) it is assumed that tasks can be processed more or less in parallel, depending on the strategy. However, the degree of parallel processing is not controlled by the relative amount of central capacity provided for each task but by the relative amount of spatial attention allocated to $S_{1}$ and $\mathrm{S}_{2}$.

\section{THE PRESENT APPROACH}

In the present study, we conducted a series of dual-task flanker experiments in which numerals served as stimuli and parity judgments were the tasks. These tasks were chosen because the corresponding S-R mapping is easy to learn and because they had already been used in similar experiments (e.g., Lien, Schweickert, \& Proctor, 2003; Logan \& Gordon, 2001; Logan \& Schulkind, 2000). In each experiment, there were single-task as well as dual-task trials. The first question was whether the relevance of the flankers for a second task increases their coprocessing during $T_{1}$. Some previous results suggest that this is indeed the case. For 
instance, Logan and Gordon (2001) conducted a dual-task flanker experiment in which, in addition to dual-task blocks, there were also single-task blocks in which a response was required only to the target $\left(S_{1}\right)$. The FCE was larger in dual tasks than in single tasks, a result that can be attributed to flankers being more intensively coprocessed on dual-task trials. In our Experiment 1, in which single and dual tasks were also blocked, we replicated this result.

A further question was whether the increased degree of flanker coprocessing in dual tasks is the result of a deliberately chosen strategy or merely the consequence of a higher memory load due to the more complex control structure necessary in dual tasks (cf. de Fockert, Rees, Frith, \& Lavie, 2001; Lavie, Hirst, de Fockert, \& Viding, 2004; Soto, Heinke, Humphreys, \& Blanco, 2005). To equate load effects, we mixed single and dual tasks in all subsequent experiments. Whether a task was also required for the flankers was indicated by a cue that was not available before the appearance of the flankers. Thus, at the beginning of each trial, participants had to be prepared in the same way. The degree of flanker coprocessing could, in principle, be adjusted only after the cue was presented. Thus, a different degree of flanker coprocessing in single and dual tasks would indicate that participants adapt their processing "on the fly" on each trial. That this is indeed the case is shown in Experiments $2 \mathrm{~A}, 2 \mathrm{~B}$, and 2C, in which the effect of different cue types was also examined.

The results of Experiments $1-2 \mathrm{C}$ raised the question of why participants coprocessed the flankers to a larger extent in dual tasks than in single tasks. For single tasks (e.g., the standard flanker task), it is obvious that the suppression of flanker processing is advantageous. Under dual-task conditions, this would imply serial processing of the stimuli for the tasks (e.g., Logan \& Gordon, 2001; Tombu \& Jolicœur, 2003). However, it is conceivable that a serial strategy might have been difficult to realize in our dual tasks, because for $T_{2}$ it would have required participants to process previously inhibited stimuli. That such processing is costly has been shown in studies of negative priming (e.g., Tipper, 1985; Tipper \& Cranston, 1985) and inhibition of return (e.g., Posner \& Cohen, 1984; Tipper, Weaver, Jerreat, \& Burak, 1994).

Thus, so as to prevent a strenuous processing of inhibited flankers, participants might process the target and flankers in parallel. Experiment 3 shows that a parallel strategy is chosen even when the costs of parallel processing for $T_{1}$ are increased by always having the flankers presented simultaneously with the target. Only when totally irrelevant flankers were presented at the beginning of a trial, in Experiment 4, did participants use a serial stimulus processing strategy for the dual tasks. Serial processing also took place in the dual tasks of Experiment 5, in which single tasks were required on $80 \%$ of the trials.

\section{EXPERIMENT 1}

In our first experiment, single and dual tasks were blocked. Single-task blocks corresponded to the standard flanker paradigm, except that the flankers appeared at different SOAs. The flankers could be congruent, incongruent, or neutral. In dual-task blocks, the same stimuli were presented as in single-task blocks, but a response was also required to the flankers (with the exception of neutral trials). Because the flankers remained on display until a participant's last response $\left(\mathrm{R}_{2}\right)$, the participants could, in principle, ignore the flankers on dual-task trials during $T_{1}$. In this case, there should be the same small FCE as on single-task trials. However, we expected that the participants would coprocess the flankers during $\mathrm{T}_{1}$ to a larger extent on dual-task trials than on single-task trials. Accordingly, the FCE should be larger for dual tasks than for single tasks.

We further hypothesized that flanker coprocessing would also produce some unspecific costs for $T_{1}$. Therefore, performance for the target should generally be impaired on dual-task trials relative to single-task trials. Moreover, on dual-task trials, performance for congruent flankers should be reduced as compared with performance for neutral ones. In the standard flanker task, performance on congruent trials is usually similar to that on neutral trials. One possible explanation is that the processing of task-relevant flankers generally produces more costs than the processing of neutral ones but that these additional costs are counterbalanced by positive response priming in case of a congruent flanker. Thus, if flankers are coprocessed to a relatively large degree, as might be the case on dual-task trials, then it could be that the increased costs of coprocessing are not outweighed by response priming. Consequently, $\mathrm{RT}_{1}$ could be larger for congruent than for neutral flankers. Such an effect would be similar to the task-set cuing effect observed in task-shift research (Rogers \& Monsell, 1995; Steinhauser \& Hübner, in press). Finally, with respect to $\mathrm{R}_{2}$, we expected the usual PRP effect.

\section{Method}

\section{Participants}

Eight students ( 3 male, 5 female; mean age $=24.0$ years) participated in the experiment either for partial fulfillment of course requirements or payment of $€ 5$ (U.S.\$6.50) per hour. All were right-handed (by self-report) and had normal or corrected-to-normal vision.

\section{Apparatus and Stimuli}

The stimuli were presented on a 21-in. (53.34-cm) color monitor (Sony 500 PS) connected to a PC, which served to control stimulus presentation and register responses.

The numerals $1,2,3,4,6,7,8$, and 9 served as targets for $\mathrm{T}_{1}$. The same set of numerals plus the neutral symbols $\%$ and \# were used as flankers. Stimuli were presented in white on a black background. The height of the stimuli subtended a visual angle of $2^{\circ}$ at a viewing distance of $110 \mathrm{~cm}$, and their width was about $1.36^{\circ}$, depending on the specific digit or symbol. $\mathrm{S}_{1}$ was presented at the center of the screen. $\mathrm{S}_{2}$ consisted of two copies of a numeral or a neutral symbol, presented on the left and right of $S_{1}$. The inner edge of each flanker was at an eccentricity of $1.18^{\circ}$.

\section{Procedure}

The task for participants was to judge the parity (odd or even) of the numerals. They had to press a left key for even and a right key for odd. Each trial started with the appearance of a fixation cross for $400 \mathrm{~ms}$. After a 600-ms blank screen, the target was presented. The flankers appeared with an SOA of $0,50,100,150,200$, or $350 \mathrm{~ms}$. The stimuli remained on the screen until $R_{2}$ on a trial. One $s$ after $R_{2}$, the cue for the next trial appeared. The flankers were neutral on $25 \%$ of the trials. On half of the remaining trials, the flankers were congruent (i.e., had the same parity as the target), and on the other half, they were incongruent (i.e., had the opposite parity). Target and flankers were always different. Errors were indicated by auditory signals. 
There were two block types: single-task and dual-task blocks. In the single-task blocks, a response was required only to the target. Responses to the target always had to be given by pushing one of two buttons with the index or middle finger of the left hand. In the dual-task blocks, participants had to respond first to the target, as on single-task trials, and subsequently to the flankers by pushing one of two other buttons with the index or middle finger of the right hand. Because no second response was necessary for neutral flankers, $25 \%$ of the trials in the dual-task blocks were actually single-task trials. After a training phase (in which one block of each trial type was performed), eight blocks of 96 trials were run alternately for each block type (eight single task and eight dual task) in two separate 1-hr sessions. The training session and the main part of the study always started with a single-task block.

\section{Results}

\section{Response Times}

$R T_{1}$

The latencies of correct responses to the target were entered into a repeated measures analysis of variance (ANOVA) with task mode (single task or dual task), congruency (congruent or incongruent), and SOA $(0,50,100,150,200$, or $350 \mathrm{~ms})$ as factors. The data for trials with neutral flankers were analyzed separately.

There was a significant main effect of task mode, $F(1,7)=$ $48.30, p<.001$. On average, the responses to the target were 384 ms longer in dual-task than in single-task blocks. Furthermore, the Task Mode $\times$ Congruency interaction was significant, $F(1,7)=$ $17.90, p<.01$. To examine this interaction in detail, we analyzed single-task and dual-task data separately.

Single task. The analysis revealed a significant Congruency $\times$ SOA interaction, $F(5,35)=5.42, p<.001$. As can be seen in Figure 1, a significant FCE (20 ms) was present only at an SOA of $0 \mathrm{~ms}, F(1,7)=15.50, p<.01$.

Dual task. In the dual-task blocks, there was a significant main effect of congruency $(144 \mathrm{~ms}), F(1,7)=23.00, p<.01$. More-

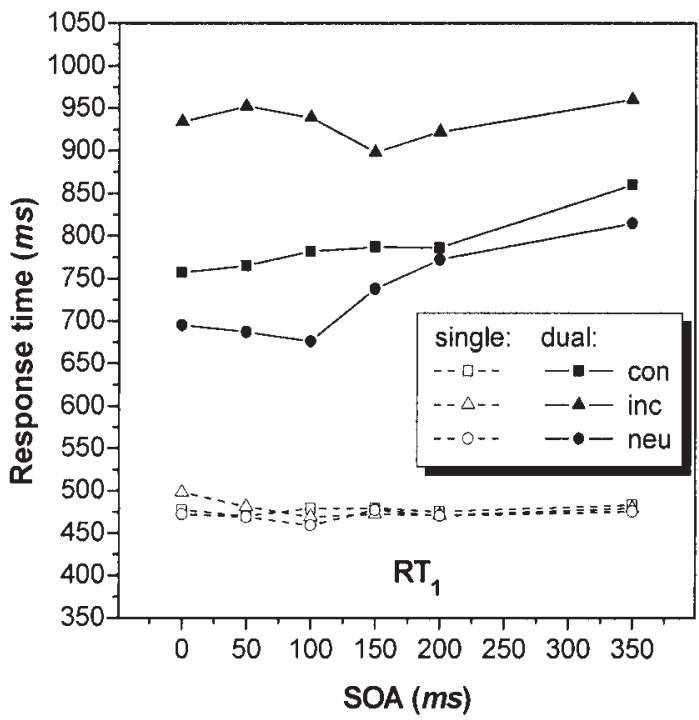

over, there was a significant Congruency $\times$ SOA interaction, $F(5$, $35)=3.67, p<.01$, indicating that the effect of congruency decreased with increasing SOA.

Neutral. An ANOVA on the latencies for neutral single-task and dual-task trials revealed significant main effects of task mode, $F(1,7)=43.20, p<.001$, and SOA, $F(5,35)=9.48, p<.001$. Responses were $261 \mathrm{~ms}$ slower on neutral trials within dual-task blocks than within single-task blocks. Furthermore, there was a significant Task Mode $\times$ SOA interaction, $F(5,35)=7.60, p<$ .001 . Response times increased with increasing SOA in dual-task blocks, but they remained constant in single-task blocks.

A further analysis revealed that latencies on neutral trials did not differ significantly from those on congruent trials in either singletask or dual-task blocks. However, as can be seen in Figure 1, on dual-task trials there was a trend in the direction of congruent stimuli producing slower responses than neutral ones, $F(1,7)=$ $3.32, p=.111$.

\section{$R T_{2}$}

The latencies of correct responses $\left(\mathrm{R}_{1}\right.$ and $\left.\mathrm{R}_{2}\right)$ to the flankers were entered into a repeated measures ANOVA with congruency (incongruent or congruent) and SOA (0, 50, 100, 150, 200, or 350 $\mathrm{ms})$ as factors. The analysis revealed a significant main effect $(212$ $\mathrm{ms}$ ) of congruency, $F(1,7)=44.30, p<.001$. There was also a significant main effect of SOA, $F(5,35)=36.90, p<.001$, reflecting a PRP effect (see Figure 1). However, there was also a significant SOA $\times$ Congruency interaction, $F(5,35)=5.12, p<$ .01 . As can be seen in Figure 1, the congruency effect decreased slightly with increasing SOA.

\section{Error Rates}

The mean error rate for $\mathrm{R}_{1}$ was $2.96 \%$, and that for $\mathrm{R}_{2}$ (given that $R_{1}$ had been correct) was $4.31 \%$. Error rates were entered into

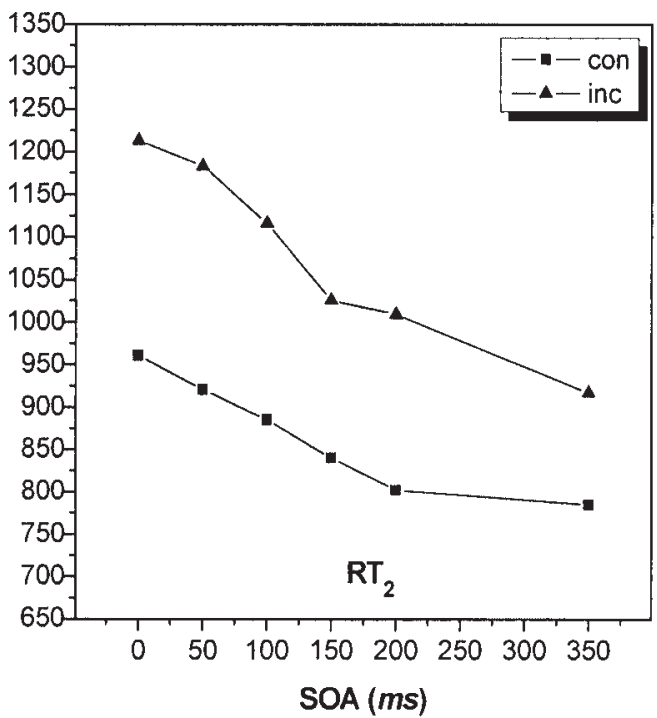

Figure 1. Response time as a function of stimulus onset asynchrony ( $\mathrm{SOA})$ for Task $1\left(\mathrm{RT}_{1}\right)$ and Task $2\left(\mathrm{RT}_{2}\right)$ in Experiment 1. single $=$ single task; dual $=$ dual task; con $=$ congruent; inc $=$ incongruent; neu $=$ neutral. 
ANOVAs that were analogous to those performed for latencies. For $\mathrm{R}_{1}$, there was a significant main effect of $\operatorname{SOA}, F(5,35)=$ $3.59, p<.05$. The error rate decreased with increasing SOA $(4.49 \%$ at $0 \mathrm{~ms} ; 1.89 \%$ at $350 \mathrm{~ms})$.

There was also a Congruency $\times$ SOA interaction, $F(5,35)=$ 2.97, $p<.05$, indicating a decreasing FCE with increasing SOA. For $\mathrm{R}_{2}$, there was a significant Congruency $\times$ SOA interaction in the same direction, $F(5,35)=2.64, p<.05$. For neutral flankers, there were no significant effects.

\section{Discussion}

The results clearly show that the degree of flanker coprocessing strongly depended on the relevance of the flankers for a subsequent task. In single-task blocks, the FCE was small $(20 \mathrm{~ms})$ and only present when the flankers were presented simultaneously with the target. This result is similar to the findings of other studies in which SOA has been varied (e.g., C. W. Eriksen \& Schultz, 1979; Flowers, 1990). In dual-task blocks, however, the FCE amounted to an average of $144 \mathrm{~ms}$. Although the effect decreased with increasing SOA, it was still substantial even at the longest SOA of $350 \mathrm{~ms}$, as can be seen in Figure 1. This large FCE, and its protracted time course, indicate that flanker processing had already started before the response selection for the target was finished. This interpretation is also supported by the trend, on dual-task trials, for neutral flankers to produce faster responses than congruent ones. There was also a large congruency effect for $\mathrm{RT}_{2}$, which decreased by about $80 \mathrm{~ms}$ with increasing SOA.

It should be noted that our results are slightly different from those obtained by Logan and Gordon (2001), who also compared single-task and dual-task performance. They observed no congruency effect for single tasks and a small effect $(29 \mathrm{~ms})$ for dual tasks. However, in an earlier study with similar stimuli and procedures, but without single-task conditions, Logan and Schulkind (2000) found a congruency effect for dual tasks that was more than three times as large as that found by Logan and Gordon and, thus, of a similar size to ours.

If we compare single and dual tasks, it is obvious that the performance was generally impaired for dual tasks. On average, $\mathrm{RT}_{1}$ was $384 \mathrm{~ms}$ longer in dual tasks than in single tasks. This seems to indicate that the increased coprocessing of flankers produced some unspecific costs. However, the size of the effect suggests that other factors contributed to the performance difference as well. First of all, it is likely that an increased memory load generally slowed processing (De Jong, 1995). This hypothesis is also supported by the results for neutral flankers. For neutral trials, only a single response was always required, even when these trials were presented within dual-task blocks. Nevertheless, there was a remarkable difference in performance between single-task and dual-task blocks, with response times on trials with neutral flankers being $261 \mathrm{~ms}$ longer in dual-task than in single-task blocks. Any form of congruency cannot account for this difference, because no response was assigned to the neutral flankers.

In addition to an effect of memory load, another factor seems important. If we again consider the performance on trials with neutral flankers, it turns out that the latencies increased by $120 \mathrm{~ms}$ over the SOA range in dual-task blocks, whereas there was no increase for single-task blocks. This could indicate that on dualtask trials, participants did not fully process $S_{1}$ from the beginning but, rather, waited (at least to some extent) for the arrival of $\mathrm{S}_{2}$. Alternatively, response-preparation effects may have been responsible. In dual-task blocks, participants were always prepared for a second response. If neutral flankers signaled that no $R_{2}$ was required, then preparation could have been abandoned or reduced. The earlier this happened, the more capacity would have been available for the processing of $T_{1}$. Presumably both waiting and response preparation played a role in the present results.

Finally, it is possible that response grouping also contributed to the performance difference between single-task and dual-task blocks. That is, participants might have withheld their response to the target until the response to the flankers was selected and then executed both responses in rapid succession. In this case, one would expect $\mathrm{RT}_{1}$ to increase with SOA (cf. Pashler, 1984; Pashler \& Johnston, 1989). Indeed, inspection of Figure 1 reveals that $\mathrm{RT}_{1}$ performance did increase slightly, at least for congruent flankers. Although this increase could also be attributed to a waiting strategy, it is hard to exclude the possibility of an effect of response grouping. To assess the extent of response grouping, we examined the interresponse intervals (IRIs). It turned out that the mean IRI was $252 \mathrm{~ms}$, whereas it should have been close to zero if response grouping had been taking place (cf. Pashler, 1984, 1994; Pashler \& Johnston, 1989). Also, the idea that the participants inserted a fixed delay between $R_{1}$ and $R_{2}$ is incompatible with the data. First, the mean IRIs for the congruent (221 ms) and incongruent conditions (291 ms) differed significantly, $F(1,7)=9.15, p<.05$. Second, the respective standard deviations of the IRIs (143 ms and $181 \mathrm{~ms}$ ) were also relatively large. Altogether, these results do not strongly support response grouping. However, if no grouping had occurred, the mean IRIs should have been somewhat longer. Thus, it seems that the participants adopted a mixture of processing strategies.

As expected, the responses $\left(R_{2}\right)$ to the flankers $\left(S_{2}\right)$ showed the usual PRP effect. Latencies decreased by $159 \mathrm{~ms}$ for congruent and by $204 \mathrm{~ms}$ for incongruent trials from an SOA of $0 \mathrm{~ms}$ to one of $200 \mathrm{~ms}$. Even though this effect alone does not imply a central bottleneck, it at least indicates some kind of central limitation (cf. Tombu \& Jolicœur, 2003).

Altogether, our considerations suggest that the large performance differences between single and dual tasks in the present experiment were likely a result of several factors. Nevertheless, the FCEs and other effects indicate that the flankers were coprocessed with the target to a larger degree in dual-task than in single-task blocks. The question is why this was the case. A possible explanation is that this reflects the applications of different strategies. However, it is also possible that the unspecific decrease in $T_{1}$ performance, as well as the increase of the specific FCE, was the result of the greater memory load in dual tasks (De Jong, 1995). With respect to the FCE, it has been shown that a high memory load reduces spatial selectivity (de Fockert et al., 2001; Lavie et al., 2004). Thus, the increased load, attributable to the more complex control structure in dual tasks, could have prevented the suppression of flanker processing during $\mathrm{T}_{1}$. To see whether this was the case, we mixed single-task and dual-task trials in the following experiments.

\section{EXPERIMENT 2A}

In this experiment, single and dual tasks were mixed within a block of trials. Mixing of trial types has often been used to 
investigate strategic versus nonstrategic components of behavior, as, for instance, in studies of the Simon effect (e.g., Marble \& Proctor, 2000; Proctor \& Vu, 2002; Proctor et al., 2003; Vu \& Proctor, 2004). With mixed trial types, however, participants have to be informed about what to do on the current trial. Because we wanted our participants to be in the same state of preparedness at the beginning of target processing on every trial, no cue was provided before the flankers appeared. Rather, a cue at flanker onset indicated whether a response was also required to the flankers. Thus, participants started each trial with the same complex mental control structure in working memory. Compared with the dual tasks in the previous experiment, the control structure for the present situation was even more complex, because it also had to include the instructions for processing the cue and for the decision of whether to process and respond to the flankers.

If high memory load on dual tasks reduced stimulus selectivity in the previous experiment (cf. de Fockert et al., 2001; Lavie et al., 2004), and if this led to the increased coprocessing of the flankers, then the FCE for single tasks should now be as large as that for dual tasks. However, if the performance differences were a result of different strategies for single and dual tasks, then there are several possibilities, depending on the adaptability of the strategies. For instance, it is possible that the degree of flanker processing can only be determined ahead of a trial. In this case, participants would have to use the same strategy for all trials. Nevertheless, they could choose $a$ particular strategy. For example, it is conceivable that under mixed conditions, participants are encouraged to suppress flanker coprocessing on dual-task trials, especially if single tasks are required on the majority of trials (because of the neutral flankers on dual-task trials). In this case, the corresponding FCEs should be as small as those for the single tasks in Experiment 1. Alternatively, participants could decide to coprocess the flankers in single tasks to the same degree that they did in the dual tasks in Experiment 1, or they could choose a strategy that lies between the two extremes. In all of these cases, though, the FCEs should not differ between single-task and dualtask trials.

If the FCE differs between single and dual tasks, this would indicate that the strategies are more flexible. Such a difference should be possible only if the degree of flanker coprocessing can be adjusted on the fly on each trial according to the cue information. We also hoped that by not providing precues and by requiring only one response on the majority of trials, we would discourage participants from any form of response grouping.

Because it is likely that spatial attention is involved in controlling the degree of flanker coprocessing (cf. Logan \& Gordon, 2001), the possibility that the cuing method itself has a great influence on performance cannot be excluded. Therefore, we applied different cuing procedures in Experiments 2A, 2B, and 2C. In Experiment 2A, the color of the flankers was used to indicate the trial type. In Experiment 2B, a color match between flankers and target was used as the cue. In Experiment $2 \mathrm{C}$, the target color signaled whether a single task or dual tasks were required.

\section{Method}

Eight students ( 7 female, 1 male; mean age $=21.5$ years) participated in the experiment either for partial fulfillment of course requirements or payment of $€ 5$ (U.S.\$6.50) per hour. All were right-handed (by self-report) and had normal or corrected-to-normal vision. The stimuli and procedure were similar to Experiment 1. Here, however, dual-task and single-task trials were mixed. To indicate the trial type, the flankers were red on $50 \%$ of the trials and green on the remaining trials, whereas the target was always white. For half of the participants, red or green flankers indicated a single task or dual tasks, respectively, whereas for the other participants, the relation was reversed. On $25 \%$ of the single-task and dual-task trials, neutral flankers ( $\%$ and \#) were presented, which were colored accordingly.

\section{Results}

\section{Response Times}

\section{$R T_{1}$}

The latencies of correct responses to the target were entered into a repeated measures ANOVA with task mode (single task or dual task), congruency (congruent or incongruent), and SOA (0, 50, $100,150,200$, or $350 \mathrm{~ms}$ ) as factors. The data for trials with neutral flankers were analyzed separately.

There was a significant main effect of task mode, $F(1,7)=$ 21.90, $p<.01$. Responses on dual-task trials were $125 \mathrm{~ms}$ slower compared with those on single-task trials. However, there was also a significant Task Mode $\times$ SOA interaction, $F(5,35)=13.20, p<$ .001 . As can be seen in Figure 2, this interaction indicates that the difference between single and dual tasks decreased with increasing SOA. Furthermore, there was a significant Task Mode $\times$ Congruency interaction, $F(1,7)=5.88, p<.05$. We examined these interactions in more detail by analyzing the task-mode conditions separately.

Single task. The analysis revealed no significant effect of congruency $(6 \mathrm{~ms}), F(1,7)=2.33, p=.171$. There was only a significant main effect of SOA, $F(5,35)=4.22, p<.01$. Response times increased by $46 \mathrm{~ms}$ across the SOA range.

Dual task. For the dual-task condition, there was a significant effect of congruency $(66 \mathrm{~ms}), F(1,7)=7.10, p<.05$. There was also a significant main effect of SOA, $F(5,35)=3.53, p<.50$. Response times decreased by about $76 \mathrm{~ms}$ over the SOA range. The Congruency $\times$ SOA interaction was marginally significant, $F(5,35)=2.47, p=.051$. The FCE was $92 \mathrm{~ms}$ at an SOA of $0 \mathrm{~ms}$ and decreased to $13 \mathrm{~ms}$ at an SOA of $350 \mathrm{~ms}$.

Neutral. The analysis for the neutral condition revealed a significant main effect of task mode, $F(1,7)=13.20, p<.01$. When the color of the flankers signaled a dual task, the latencies were $14 \mathrm{~ms}$ longer relative to single-task trials. Furthermore, the main effect of SOA was significant, $F(5,35)=7.75, p<.001$. Response times increased by about $59 \mathrm{~ms}$ over the SOA range.

The performance of neutral and congruent flankers differed significantly for single-task, $F(1,7)=13.90, p<.01$, and dualtask trials, $F(1,7)=30.20, p<.001$. In each case, neutral flankers produced faster responses than congruent ones $(16 \mathrm{~ms}$ for single tasks; $97 \mathrm{~ms}$ for dual tasks).

$R T_{2}$

The latencies of correct responses $\left(\mathrm{R}_{1}\right.$ and $\left.\mathrm{R}_{2}\right)$ to the flankers were entered into a repeated measures ANOVA with congruency (incongruent or congruent) and SOA $(0,50,100,150,200$, or 350 $\mathrm{ms})$ as factors. The analysis revealed a significant main effect of 

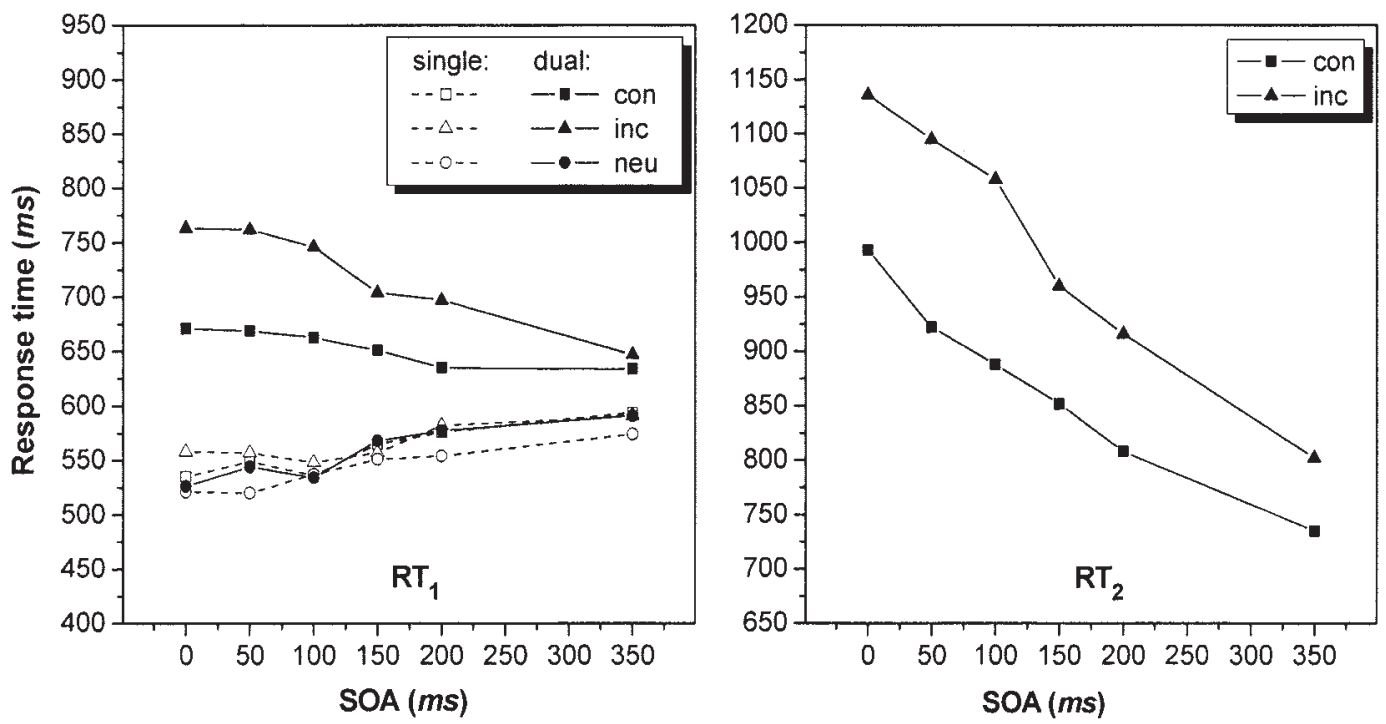

Figure 2. Response time as a function of stimulus onset asynchrony (SOA) for Task $1\left(\mathrm{RT}_{1}\right)$ and Task $2\left(\mathrm{RT}_{2}\right)$ in Experiment 2A. single = single task; dual $=$ dual task; con = congruent; inc $=$ incongruent; neu $=$ neutral .

congruency, $F(1,7)=10.80, p<.05$. Furthermore, there was a significant effect of SOA, $F(5,35)=62.20, p<.001$, indicating a PRP effect. However, there was also a reliable SOA $\times$ Congruency interaction, $F(5,35)=4.95, p<.01$. The congruency effect decreased with increasing SOA.

\section{Error Rates}

The mean error rate for $\mathrm{R}_{1}$ was $1.93 \%$, and that for $\mathrm{R}_{2}$ (given that $\mathrm{R}_{1}$ had been correct) was $5.89 \%$. The difference in error rate between single $(2.34 \%)$ and dual tasks $(1.51 \%)$ for $\mathrm{R}_{1}$ was significant, $F(1,7)=18.10, p<.01$. Furthermore, there was a significant main effect of congruency, $F(1,7)=5.83, p<.05$, indicating that more errors occurred with incongruent flankers. Additionally, SOA produced a significant main effect, $F(5,35)=$ $4.34, p<.05$. However, there was also a Task Mode $\times$ SOA interaction, $F(5,35)=2.55, p<.05$, indicating that the error rate was highest for single-task trials with short SOAs. As far as the neutral trials are concerned, there was only a significant main effect of task mode, $F(1,7)=6.24, p<.05$. More errors occurred for single tasks. For $\mathrm{RT}_{2}$, no factor produced a significant effect.

\section{Comparison With Experiment 1}

To compare performance between Experiment 1 and Experiment $2 \mathrm{~A}$, we computed several ANOVAs. An ANOVA for $\mathrm{R}_{1}$ with the between-participants factor of experiment (1 or 2A) and the within-participant factors of congruency (incongruent or congruent), SOA $(0,50,100,150,200$, or $350 \mathrm{~ms})$, and task mode (single task or dual task) revealed a significant Experiment $\times$ Task Mode interaction, $F(1,14)=17.90, p<.001$. The response time difference between single-task and dual-task trials was much larger in Experiment 1 (384 ms) than in Experiment 2A (125 ms). Furthermore, the Experiment $\times$ Task Mode $\times$ SOA interaction was significant, $F(5,70)=10.10, p<.001$. On dual-task trials, the overall latencies decreased in Experiment 2A but increased in Experiment 1.

To examine the Task Mode $\times$ Experiment interaction, we analyzed the data for single-task and dual-task trials separately. There was a significant main effect of experiment for single-task trials, $F(1,14)=5.99, p<.05$. Latencies were $84 \mathrm{~ms}$ shorter in Experiment 1 than in Experiment 2A. The opposite was the case for dual-task trials, $F(1,14)=5.51, p<.05$. Here, the latencies were $175 \mathrm{~ms}$ longer in Experiment 1. The congruency effect on single-task trials did not differ significantly between the experiments, $F(1,14)=0.71, p=.413$. For dual-task data, the Congruency $\times$ Experiment interaction was marginally significant, $F(1$, $14)=4.08, p=.063$. The FCE was $66 \mathrm{~ms}$ in Experiment $2 \mathrm{~A}$ and $144 \mathrm{~ms}$ in Experiment 1.

Comparison of neutral trials revealed a significant Experiment $\times$ Task Mode interaction, $F(1,14)=38.30, p<.001$. The difference between single tasks and dual tasks was much larger in Experiment 1 (261 ms) than in Experiment 2A (14 ms). Additionally, there was a reliable Experiment $\times$ Task Mode $\times$ SOA interaction, $F(5,70)=4.97, p<.001$. For Experiment 1, dual-task latencies increased over the SOA range, whereas single-task latencies remained constant. In Experiment 2A, latencies increased by about the same degree for single and dual tasks.

With respect to $\mathrm{RT}_{2}$, there were no significant differences between the experiments.

\section{Discussion}

In the present experiment, single-task and dual-task trials were mixed within a block of trials, and there was no precue that would have allowed the participants to prepare in advance for a specific type of trial. With a control structure that merely controls whether $\mathrm{R}_{2}$ is executed and otherwise puts the same stimulus processing into operation on each trial, similar FCEs for single and dual tasks should have occurred. However, if the degree of flanker processing 
was adapted on each trial according to the trial type, then the corresponding FCEs should have differed.

As the results show (see Figure 2), the latter was the case. The FCE was significantly larger for dual tasks than for single tasks. However, compared with Experiment 1, the FCE for dual tasks was smaller here (66 ms vs. $144 \mathrm{~ms}$ ). This suggests that the flankers on dual-task trials were coprocessed less intensively compared with those in the previous experiment. Moreover, the overall $\mathrm{RT}_{1}$ difference between single and dual tasks was reduced under randomized (125 ms) as compared with blocked conditions (384 ms). Relative to Experiment 1, participants were slower on singletask trials (by $84 \mathrm{~ms}$ ), which was presumably a result of the more complex control structure. Because of the mixed trial types, the participants had to be prepared for dual tasks on each trial, which probably led to the impairment on single-task trials. The same reasoning holds for the impaired performance on neutral trials. However, responses on dual-task trials were faster (by $175 \mathrm{~ms}$ ) relative to those in the first experiment.

It is interesting to note that the speedup on dual-task trials relative to Experiment 1 was twice as large as the slowing on single-task trials. There are several possible reasons. First of all, presumably due to the mixing of single and dual tasks, there was no strong indication of response grouping in Experiment 2A (the mean IRI was $385 \mathrm{~ms}$ ). Clearly, because the flankers were task irrelevant on $62.5 \%$ of trials (single-task trials plus neutral trials), response grouping would not have made much sense. Furthermore, the high frequency of single-task trials also reduced waiting effects, as indicated by the SOA effect for neutral trials. Although the latencies on neutral trials still increased by $53 \mathrm{~ms}$ with increasing SOA, this increase was much smaller than that in Experiment 1. For congruent and incongruent trials, there was even a significant SOA effect on $\mathrm{RT}_{1}$ in the opposite direction. Latencies decreased by $76 \mathrm{~ms}$ with increasing SOA. However, this effect might be underestimated because of waiting.

With respect to $R_{2}$, we again observed a PRP effect and a significant congruency effect. Comparison with Experiment 1 revealed no significant differences with respect to $\mathrm{RT}_{2}$.

Altogether, even though the mixing of trial types reduced performance differences between single and dual tasks, the results clearly show that flankers were again processed during $T_{1}$ to a larger extent in dual than in single tasks. This raises the question of how the different processing strategies were achieved by participants. Because the trial type was not known before flanker onset, our results seem to indicate that the respective degree of flanker processing was adjusted on the fly during the processing of $\mathrm{T}_{1}$. For instance, it is conceivable that more spatial attention was dynamically allocated to the flankers on dual-task trials (cf. Logan \& Gordon, 2001). However, it is also possible that participants simply implemented a fixed, early filter mechanism. Because the flankers on single-task trials always had a certain color, color could have been used to filter out flankers. In other words, the participants might have processed flankers of a certain color and ignored flankers of a different color. That color can generally be used for stimulus selection instead of location has been demonstrated by Hübner and Backer (1999; for an early example in a flanker paradigm, see also Humphreys, 1981). Whether this was also the case in the present experiment was tested in Experiment 2B.

\section{EXPERIMENT 2B}

Whereas in the previous experiment, the color of the flankers signaled the type of trial, here trial type was signaled by whether the color (red or green) of target and flankers matched. With this procedure, the degree of flanker processing could no longer be based on a fixed, early color-filtering strategy. Consequently, a different degree of flanker coprocessing for single and dual tasks during $\mathrm{T}_{1}$ would indicate that participants used another mechanism to implement their strategies.

\section{Method}

Eight students ( 7 female, 1 male; mean age $=25.0$ years) participated in the experiment either for partial fulfillment of course requirements or payment of $€ 5$ (U.S.\$6.50) per hour. All were right-handed (by self-report) and had normal or corrected-to-normal vision. Stimuli and procedure were the same as in the previous experiment, with the exception that here the target was also colored at flanker onset. On half of the trials, targets and flankers had the same color; on the other half, they appeared in a different color. Red and green served as colors and were balanced across trials. A single-task trial was indicated by the target and flankers having the same color, whereas different colors indicated a dual-task trial. Again, on some trials in the dual-task condition, neutral flankers were presented, which were colored accordingly.

\section{Results}

\section{Response Times}

$R T_{1}$

The latencies of correct responses to the target in single-task and dual-task trials were entered into a repeated measures ANOVA with task mode (single task or dual task), congruency (congruent or incongruent), and SOA $(0,50,100,150,200$, or $350 \mathrm{~ms})$ as factors. The data for the neutral trials were analyzed separately.

The analysis revealed a significant main effect of task mode, $F(1,7)=14.80, p<.01$. On average, latencies were $90 \mathrm{~ms}$ longer on dual-task than on single-task trials. The Task Mode $\times$ Congruency interaction was marginally significant, $F(1,7)=4.10, p=$ .082 . The FCEs were $23 \mathrm{~ms}$ and $55 \mathrm{~ms}$ for single tasks and dual tasks, respectively. Further, the Task Mode $\times$ SOA interaction was significant, $F(5,35)=8.73, p<.001$. As can be seen in Figure 3, this interaction indicates that the latency difference between single-task and dual-task trials decreased with increasing SOA. To examine these interactions in more detail, we also analyzed the data for single tasks and dual tasks separately.

Single task. The ANOVA revealed significant main effects of congruency, $F(1,7)=12.50, p<.01$, and SOA, $F(5,35)=3.08$, $p<.05$. However, there was also a significant Congruency $\times$ SOA interaction, $F(5,35)=3.85, p<.01$, indicating that the FCE decreased with increasing SOA.

Dual task. There was a significant main effect of congruency, $F(1,7)=13.60, p<.01$. However, there was also a significant Congruency $\times$ SOA interaction, $F(5,35)=4.29, p<.01$, indicating stronger congruency effects at shorter SOAs.

Neutral. The analysis of the data for the neutral condition revealed a significant main effect of task mode, $F(1,7)=14.10$, $p<.01$. Responses were slower (by $16 \mathrm{~ms}$ ) on dual-task than on 

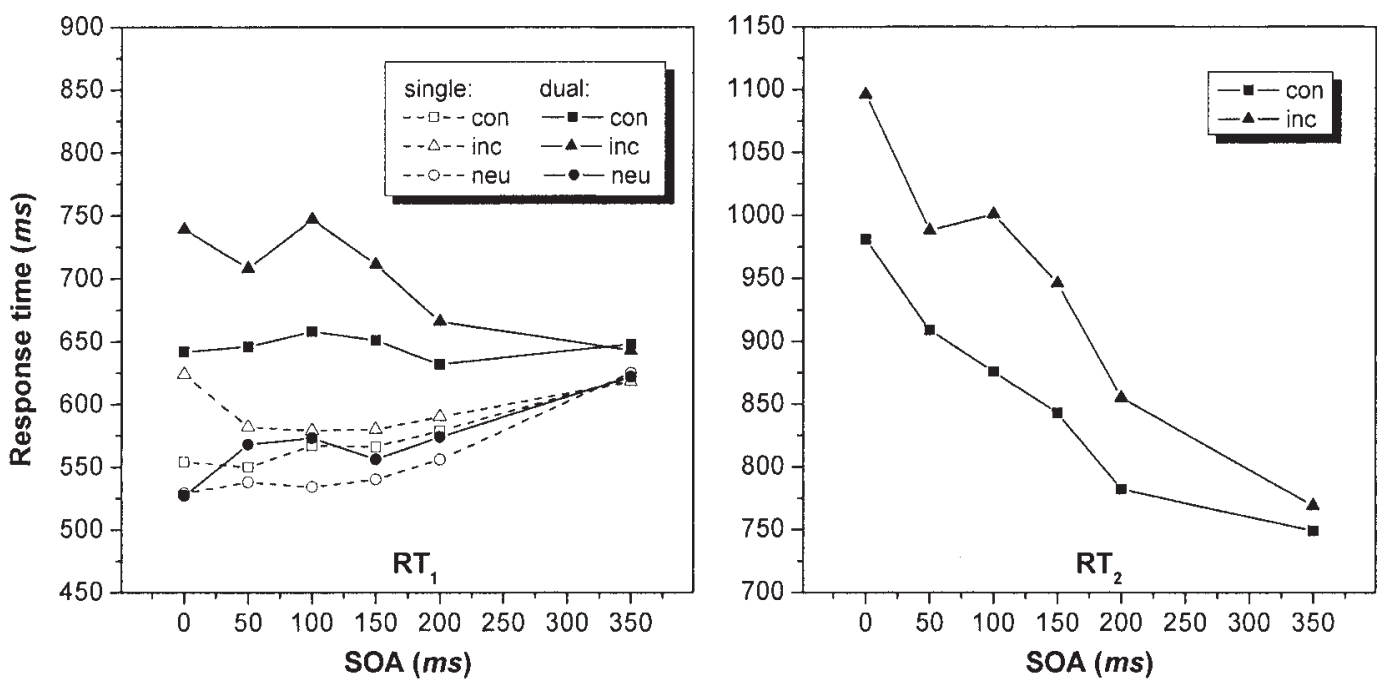

Figure 3. Response time as a function of stimulus onset asynchrony ( $\mathrm{SOA})$ for Task $1\left(\mathrm{RT}_{1}\right)$ and Task $2\left(\mathrm{RT}_{2}\right)$ in Experiment $2 \mathrm{~B}$. single $=$ single task; dual $=$ dual task; con $=$ congruent; inc $=$ incongruent; neu $=$ neutral.

single-task trials. Additionally, the latencies increased significantly with increasing SOA (by $96 \mathrm{~ms}$ ), $F(5,35)=9.00, p<.001$.

The difference between neutral and congruent trials was significant for both single-task, $F(1,7)=83.90, p<.001$, and dualtasks trials, $F(1,7)=17.60, p<.01$. Neutral flankers led to faster responses than did congruent ones (19 ms for single tasks; $77 \mathrm{~ms}$ for dual tasks).

\section{$R T_{2}$}

The latencies of correct responses $\left(R_{1}\right.$ and $\left.R_{2}\right)$ to the flankers were entered into a repeated measures ANOVA with congruency (incongruent or congruent) and SOA $(0,50,100,150,200$, or 350 $\mathrm{ms})$ as factors. The analysis revealed a significant effects of congruency $(85 \mathrm{~ms}), F(1,7)=39.50, p<.001$, and SOA, $F(5$, $35)=72.90, p<.001$. Moreover, the SOA $\times$ Congruency interaction was significant, $F(5,35)=3.68, p<.01$, indicating a decreasing congruency effect with increasing SOA.

\section{Error Rates}

The mean error rate for $R_{1}$ was $1.93 \%$, and that for $R_{2}$ (given that $\mathrm{R}_{1}$ had been correct) was $3.74 \%$. For $\mathrm{R}_{1}$, more errors were made on single-task $(2.41 \%)$ than on dual-task trials $(1.46 \%), F(1$, $7)=5.84, p<.05$. For the neutral condition, there was a significant main effect of SOA, $F(5,35)=4.35, p<.01$. More errors occurred at shorter SOAs. For $\mathrm{R}_{2}$ error rate, no factor produced significant effects.

\section{Discussion}

In this experiment, the trial type was indicated by a color match or mismatch between target and flankers. With this procedure, it was no longer possible for participants to use a simple colorfiltering mechanism to process the flankers in single and dual tasks differently. As inspection of Figure 3 shows, the results were rather similar to those of the previous experiment. The overall latencies for $\mathrm{R}_{1}$ again differed between single and dual tasks, and this difference decreased with increasing SOA. Most important, though, the FCEs still differed between single-task and dual-task trials, and they were even similar in size to those in Experiment $2 \mathrm{~A}$. The same held for $\mathrm{RT}_{2}$. The situation was slightly different for single tasks. Compared with that in the previous experiment, the FCE was larger, which was probably attributable to the fact that here, target and flankers had the same color on single-task trials, which impaired target selection (cf. Harms \& Bundesen, 1983; Kramer \& Jacobson, 1991).

Altogether, the results of the present experiment demonstrate that the FCE differences between the trial types in the previous experiment did not depend on early color-filtering mechanisms.

\section{EXPERIMENT 2C}

Experiment $2 \mathrm{C}$ examined whether the fact that the flankers always had to be attended for cue encoding in the previous two experiments favored a parallel processing strategy for dual tasks. Here, a cuing procedure was used that allowed participants to encode the cue without attending to the flankers. The color of the flankers was constantly white, whereas that of the target was also white at the beginning of a trial but then changed with flanker onset. The new target color signaled the trial type. Thus, if the requirement to process the flankers for cue encoding encouraged the participants in the previous two experiments to coprocess the flankers more in dual than in single tasks, a similarly small FCE should occur for both trial types in the present experiment. However, if the increased flanker coprocessing in dual tasks was attributable to more general factors, then a similar FCE difference as in the previous experiments should occur.

\section{Method}

Eight students ( 6 female, 2 male; mean age $=24.1$ years $)$ participated either for partial fulfillment of course requirements or payment of $€ 5$ 
(U.S.\$6.50) per hour. All were right-handed (by self-report) and had normal or corrected-to-normal vision. The stimuli and procedure were the same as in the previous experiment, with the exception that the flankers always appeared in white, and the color of the target was used as the cue. The target first appeared in white. At flanker onset, the target changed color to red or green, indicating a single- or a dual-task trial, respectively. On trials with neutral flankers, the target was also randomly colored red or green. For half of the participants, red indicated a dual-task trial and green indicated a single-task trail; for the other half, the relation was reversed.

\section{Results}

\section{Response Times}

$R T_{1}$

The latencies of correct responses to the target in single-task and dual-task trials were entered into a repeated measures ANOVA with task mode (single task or dual task), congruency (congruent or incongruent), and SOA $(0,50,100,150,200$, or $350 \mathrm{~ms})$ as factors. The data for neutral trials were analyzed separately.

The analysis revealed a significant main effect of task mode, $F(1,7)=6.21, p<.05$. On average, the latencies were $45 \mathrm{~ms}$ longer on dual-task than on single-task trials. However, there was also a significant Task Mode $\times$ Congruency interaction, $F(1,7)=$ $5.95, p<.05$. The FCEs were $10 \mathrm{~ms}$ and $43 \mathrm{~ms}$ for single and dual tasks, respectively. Furthermore, the Task Mode $\times$ SOA interaction was significant, $F(5,35)=9.96, p<.001$. As can be seen in Figure 4, the latency difference between single-task and dual-task trials decreased with increasing SOA. To examine these interactions in more detail, we also analyzed the data for single-task and dual-task trials separately.

Single task. The ANOVA revealed significant main effects of congruency, $F(1,7)=6.68, p<.05$, and SOA, $F(5,35)=7.49$, $p<.001$. The Congruency $\times$ SOA interaction was not significant, $F(5,35)=1.32, p=.275$.

Dual task. There was a significant main effect of congruency, $F(1,7)=8.94, p<.05$. Furthermore, the Congruency $\times$ SOA interaction was significant, $F(5,35)=2.85, p<.05$, indicating that the FCE decreased with increasing SOA.

Neutral. The analysis of the data for the neutral condition revealed no significant main effect of task mode, $F(1,7)=2.55$, $p=.15$. However, the effect of SOA was significant, $F(5,35)=$ $11.50, p<.001$. The latencies increased by $77 \mathrm{~ms}$ over the SOA range.

Neutral flankers led to faster responses than did congruent ones. The effect was $13 \mathrm{~ms}$ for single tasks, which was marginally significant, $F(1,7)=4.90, p=.062$, and $51 \mathrm{~ms}$ for dual tasks, which was significant, $F(1,7)=6.13, p<.05$.

$R T_{2}$

The latencies of correct responses $\left(R_{1}\right.$ and $\left.R_{2}\right)$ to the flankers were entered into a repeated measures ANOVA with congruency (incongruent or congruent) and SOA $(0,50,100,150,200$, or 350 $\mathrm{ms})$ as factors. The analysis revealed a significant effect of congruency (77 ms), $F(1,7)=5.91, p<.05$. Also, the effect of SOA was reliable, $F(5,35)=1.43, p<.001$. However, there was also a significant Congruency $\times \mathrm{SOA}$ interaction, $F(5,35)=3.43, p<$ .05 , indicating a decreasing congruency effect with increasing SOA.

\section{Error Rates}

The mean error rate for $R_{1}$ was $3.28 \%$, and that for $R_{2}$ (given that $\mathrm{R}_{1}$ had been correct) was $7.01 \%$. For $\mathrm{R}_{1}$, there was a significant effect of task mode, $F(1,7)=13.50, p<.01$. More errors occurred on single-task $(4.12 \%)$ than on dual-task trials $(2.45 \%)$. There was also a reliable effect of congruency (congruent: $2.58 \%$; incongruent: $3.99 \%), F(1,7)=13.50, p<.01$. Furthermore, there was a significant main effect of SOA, $F(1,7)=5.99, p<.001$ indicating that the error rate decreased with increasing SOA. For the neutral condition, there was a significant main effect of task mode, $F(1,7)=6.74, p<.05$, with more errors occurring on
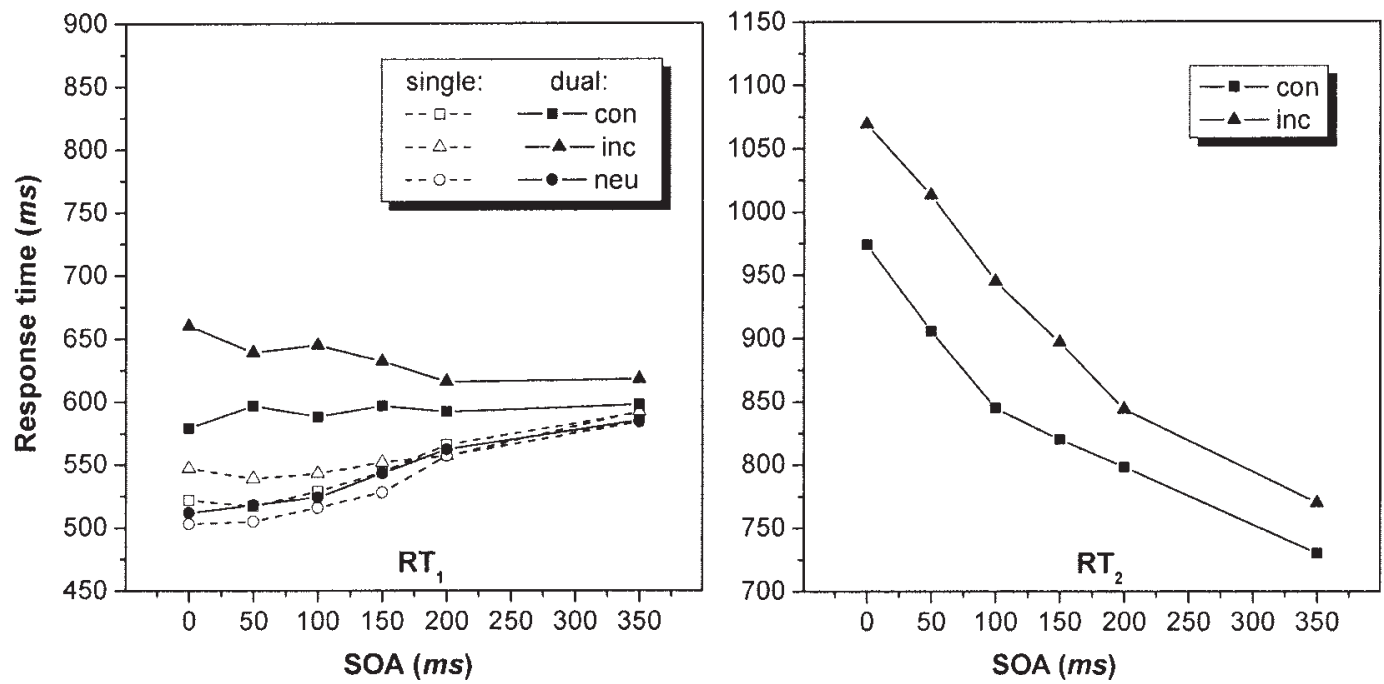

Figure 4. Response time as a function of stimulus onset asynchrony (SOA) for Task $1\left(\mathrm{RT}_{1}\right)$ and Task $2\left(\mathrm{RT}_{2}\right)$ in Experiment $2 \mathrm{C}$. single $=$ single task; dual $=$ dual task; con $=$ congruent; inc $=$ incongruent; neu $=$ neutral. 
neutral $(5.64 \%)$ than on single-task trials $(3.88 \%)$. Furthermore, there was a significant main effect of SOA, $F(5,35)=4.49, p<$ .01 . More errors occurred at shorter SOAs. For $\mathrm{R}_{2}$ error rate, no factor produced significant effects.

\section{Discussion}

Experiment $2 \mathrm{C}$ aimed to examine whether a cuing procedure that does not require participants to attend to the flankers leads to a reduced flanker coprocessing in dual tasks. Here, trial-type information was carried only by the target color that appeared simultaneously with flanker onset. As the results show, with respect to FCEs, the new cuing procedure led to a similar performance as the cuing procedures used previously. This demonstrates that the flankers were still processed to a larger degree in dual than in single tasks.

In contrast to the previous experiments, though, there was no significant effect of SOA on $\mathrm{RT}_{1}$. This could have been the result of a waiting strategy. As indicated by the increased latencies with increasing SOA for neutral flankers, the participants seem to have waited for the color change of the target. The waiting effect presumably counterbalanced an SOA effect in the opposite direction. With respect to $\mathrm{RT}_{2}$, there was again the usual PRP effect.

Altogether, the results of the Experiments 2A, 2B, and 2C demonstrate that irrespective of the specific cuing procedure, the participants generally coprocessed flankers more in dual tasks than in single tasks. The fact that this occurred even though the trial types were mixed suggests that participants adjusted the coprocessing of flankers on the fly, as a function of whether the flankers had to be used for a second task. We now turn to the question of why this was the case.

\section{EXPERIMENT 3}

In this experiment, we investigated the hypothesis that participants processed the stimuli more in parallel in dual tasks because serial processing of the target and flankers would have been rather strenuous. A serial strategy would require participants to suppress flanker processing during $T_{1}$ and then, after the selection of $R_{1}$, process these inhibited stimuli. This could be effortful, as is suggested by such phenomena as negative priming (e.g., Tipper, 1985; Tipper \& Cranston, 1985) and inhibition of return (Posner \& Cohen, 1984; Tipper et al., 1994). Therefore, participants might accept some performance costs in $\mathrm{T}_{1}$ to avoid such increased mental-control costs (cf. Logan et al., 1983). In the previous experiments, this could have especially been the case, because the costs for $T_{1}$ produced by flanker coprocessing were relatively small for two reasons. First, flankers were incongruent on only half of the trials. Second, because of the SOA variation, flankers appeared, on average, $142 \mathrm{~ms}$ after the target onset, which also reduced the costs. Clearly, the largest costs of flanker coprocessing occurred at an SOA of $0 \mathrm{~ms}$.

These considerations suggest that it might be possible to encourage participants to apply a serial strategy by further increasing the costs in performance for $\mathrm{T}_{1}$ produced by parallel processing of target and flankers. In the present experiment, we attempted to achieve this by always presenting the flankers simultaneously with the target. Moreover, we also delayed the cues. That is, after a variable period (the stimulus-cue interval $[\mathrm{SCI}]$ ), both the target and the flankers changed color. As in Experiment 2B, a color match or mismatch indicated a single task or dual tasks, respectively. Thus, although the flankers were presented together with the target, whether a response to them was also required remained unknown until their color changed.

We assessed how participants processed the flankers under these conditions. If they still avoided the suppression of flankers in dual tasks, then flanker processing would start right from flanker onset on all trials. On single-task trials, the processing could be abandoned or suppressed only after the cue appeared. If this conjecture is correct, several outcomes can be expected. First, the FCE for single-task trials should increase with increasing SCI. Second, the FCE for dual tasks should be relatively independent of the SCI. Third, the FCE for dual tasks should be similar in size to the corresponding FCEs in the previous experiments at the shortest SOA.

However, if the new procedure encouraged participants to apply a serial strategy, then flanker processing should be suppressed during $\mathrm{T}_{1}$ on all trials. In this case, the FCE for single tasks should be similar in size to the corresponding effects in the previous experiments. The outcome is less predictable for dual tasks, because there are several possibilities. For instance, flanker processing could be suppressed only until the cue appears and then start immediately if a dual task is signaled. This should lead to results similar to those of the previous experiments. However, if flanker processing remains suppressed until $\mathrm{R}_{1}$ has been selected, then the FCE should be as small as on single-task trials.

\section{Method}

Eight individuals ( 7 female, 1 male; mean age $=21$ years) participated either for partial fulfillment of course requirements or payment of $€ 5$ (U.S.\$6.50) per hour. All were right-handed (by self-report) and had normal or corrected-to-normal vision. The stimuli and procedure were similar to those of the previous experiment. Here, however, target and flankers always appeared simultaneously in white. After a certain time interval (SCI), the items changed color (at an SCI of $0 \mathrm{~ms}$, target and flankers appeared colored right from the beginning of stimulus presentation). The colors and the meaning of color matches and mismatches were the same as in Experiment 2B. After a training block, 16 blocks of 96 trials were run in two separate 1-hr sessions.

\section{Results}

\section{Response Times}

$R T_{1}$

The latencies of correct responses to the targets were entered into a repeated measures ANOVA with task mode (single-task or dual-task), congruency (congruent or incongruent), and SCI (0, 50, $100,150,200$, or $350 \mathrm{~ms}$ ). The data for the neutral trials were analyzed separately.

The analysis revealed a significant main effect of task mode, $F(1,7)=32.80, p<.001$. Latencies were $83 \mathrm{~ms}$ longer for dual-task than for single-task trials. However, there was also a significant Task Mode $\times$ Congruency interaction, $F(1,7)=6.40$, $p<.05$, indicating that the FCE was larger on dual-task (88 ms) than on single-task trials $(54 \mathrm{~ms})$. There was also a main effect of SCI, $F(5,35)=22.70, p<.001$. However, there was a significant $\mathrm{SCI} \times$ Task Mode interaction, $F(5,35)=9.34, p<.001$. For 
single tasks, the response times increased by $96 \mathrm{~ms}$ with increasing SCI, whereas the increase was only $39 \mathrm{~ms}$ for dual tasks.

Neutral. An analysis of the neutral trials revealed a significant main effect of SCI, $F(5,35)=7.03, p<.001$. Latencies increased by $76 \mathrm{~ms}$ with increasing SCI. There was no significant difference between the latencies for single and dual tasks, $F(1,7)=1.43, p=$ .270 .

Significant differences were found between neutral and congruent trials for single tasks, $F(1,7)=52.50, p<.001$, and dual tasks, $F(1,7)=82.20, p<.001$. Latencies on neutral trials were again shorter than on congruent trials (64 ms for single tasks; 125 ms for dual tasks).

\section{$R T_{2}$}

The latencies of correct responses $\left(R_{1}\right.$ and $\left.R_{2}\right)$ were entered into a repeated measures ANOVA with congruency (incongruent or congruent) and SCI $(0,50,100,150,200$, or $350 \mathrm{~ms})$ as factors. There was a significant main effect of congruency $(110 \mathrm{~ms}), F(1$, $7)=35.80, p<.01$. Moreover, the effect of SCI was reliable, $F(5$, $35)=19.40, p<.001$. Latencies increased by $114 \mathrm{~ms}$ with increasing SCI.

\section{Error Rates}

The mean error rate for $\mathrm{R}_{1}$ was $2.13 \%$, and that for $\mathrm{R}_{2}$ (given that $\mathrm{R}_{1}$ had been correct) was $3.74 \%$. The error rates for single $(2.35 \%)$ and dual tasks (1.91\%) did not differ significantly, $F(1$, $7)=3.39, p=.108$. Significantly more errors were made on incongruent $(2.53 \%)$ than on congruent trials $(1.73 \%), F(1,7)=$ 9.27, $p<.05$. Apart from that, there was a reliable effect of SCI, $F(5,35)=3.17, p<.05$. Most errors occurred at the SCIs of 50 and $350 \mathrm{~ms}$. For the neutral condition, no factor produced significant effects. This was also the case for $\mathrm{R}_{2}$ error rates.

\section{Discussion}

In the present experiment, target and flankers were presented simultaneously, whereas the cue signaling the trial type appeared at a variable SCI. This procedure should have increased the costs in performance for $\mathrm{T}_{1}$ produced by a parallel processing strategy. The main question was whether this would encourage participants to adopt a serial processing strategy in dual tasks. As our results show, this was not case (see Figure 5). Rather, the participants again applied a parallel strategy, as indicated by the large FCEs. Here, FCEs were even larger than those in the previous experiments, mainly due to the fact that the FCEs remained relatively constant in size across the SCIs. This was the case not only for dual tasks but for single tasks as well. Furthermore, the FCE was again larger in dual tasks than in single tasks. It is interesting to note that the mean FCEs for the two trial types were similar in size to the corresponding FCEs in the equivalent condition in Experiment $2 \mathrm{~B}$ at the shortest SOA. This suggests that participants immediately coprocessed the flankers when they appeared, at least to some degree and for some time, and that this is why the FCE was relatively unaffected by the SCI.

A further mechanism apparent in the present data relates to the dynamics of preparation for the second response (cf. De Jong, 1995). It seems that the participants, presumably encouraged by the already available flankers, had prepared for $\mathrm{R}_{2}$ to some extent before the cue appeared. If the cue then signaled a single task, the preparation process was stopped. The earlier this happened, the more resources were available for the processing of $T_{1}$, which explains why $\mathrm{RT}_{1}$ increased with an increasing SCI on single-task trials (see Figure 5). However, if the cue signaled a dual-task trial, then preparation was intensified. The earlier this occurred, the better participants were prepared, which is reflected in the decreasing $\mathrm{RT}_{2}$ with a decreasing SCI. Because this intensified preparation took place anyway on dual-task trials, there was no substantial SCI effect on $\mathrm{RT}_{1}$. Furthermore, this extra preparation might also have increased the memory load as a result of the stronger activation of a more complex control structure (cf. de Fockert et al., 2001; Lavie et al., 2004), and this could account for the increase in the FCE relative to single tasks.

What can definitively be ruled out by our data is the possibility that the flankers were coprocessed on single-task trials only until
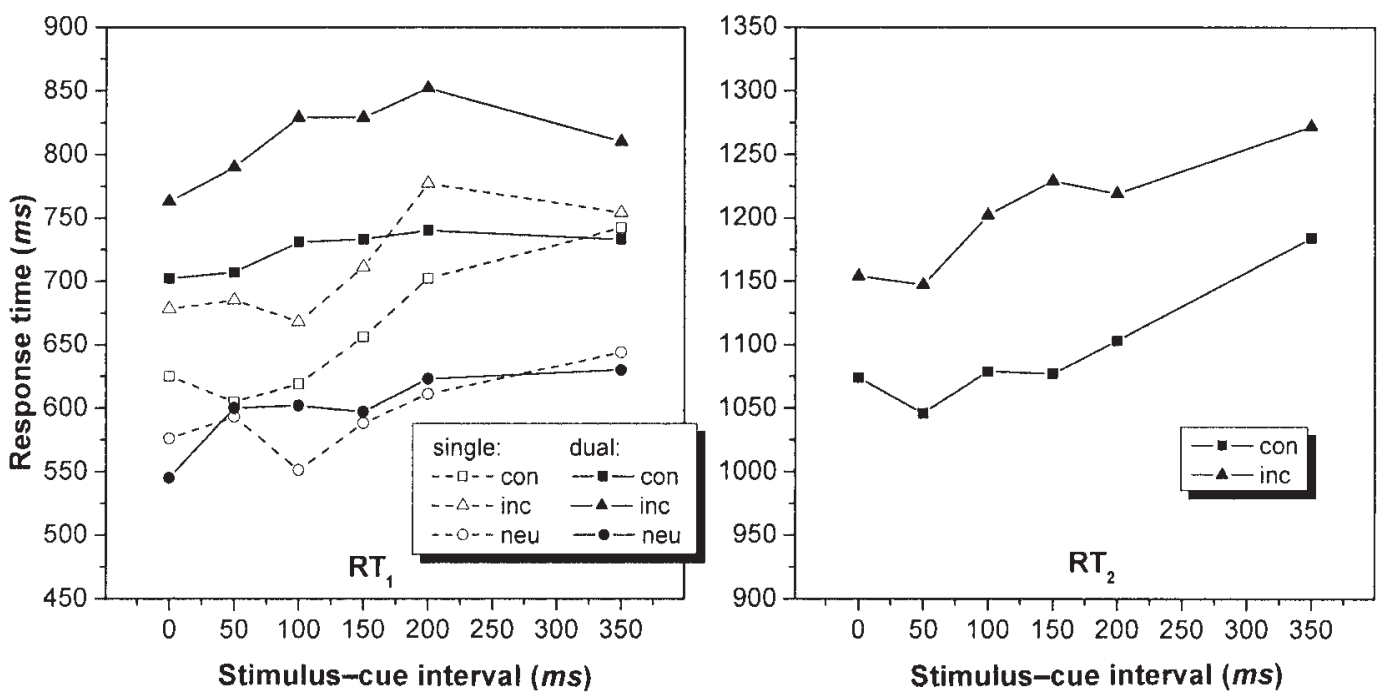

Figure 5. Response time as a function of stimulus-cue interval for Task $1\left(\mathrm{RT}_{1}\right)$ and Task $2\left(\mathrm{RT}_{2}\right)$ in Experiment 3 . single $=$ single task; dual $=$ dual task; con $=$ congruent; inc $=$ incongruent; neu $=$ neutral. 
the cue arrived. If this had been the case, then the FCE should have increased with increasing SCI, which is not what we found. Rather, it seems that the flankers were coprocessed to a certain extent irrespective of when the cue arrived, and merely the preparedness for the second task varied with the SCI. The fact that the degree of flanker coprocessing was relatively constant, even on single-task trials, suggests that it is indeed difficult or even impossible to change a once-chosen strategy for flanker coprocessing, at least within a certain time window.

A final aspect of the data concerns the latencies observed for neutral flankers. Neutral flankers provide a good opportunity to disentangle the effects of preparedness and waiting. In Experiment 1 , it remained an open question whether the increase in $\mathrm{RT}_{1}$ with increasing SOA was a result of waiting for the flankers. Here, the neutral flankers always appeared simultaneously with the target and, thus, immediately signaled that no second response was required. Nevertheless, $\mathrm{RT}_{1}$ still increased by $76 \mathrm{~ms}$ across the SOAs. This implies that participants have the general strategy to wait for some time for the arrival of the cue, even though the cue is uninformative on trials with neutral flankers.

Taken together, the results clearly demonstrate that the participants used a parallel processing strategy on dual tasks, even under the current conditions. This is striking, because the strategy produced a large amount of interference and, therefore, costs in performance for $T_{1}$. From this one might wonder whether participants are capable of ignoring $\mathrm{S}_{2}$ flankers on dual-task trials. Is there any way to encourage participants to adopt a serial strategy in such situations? This was tested in Experiments 4 and 5.

\section{EXPERIMENT 4}

In this experiment, we used a specific procedure to examine whether a serial strategy is possible on dual-task trials. As in Experiment 3, the target and flankers were presented in white at the same time. Also, after a variable period of time, both target and flankers were colored to indicate the trial type. The crucial difference here was that the flankers also changed their identity with changed coloring, whereas the target's identity remained the same. Thus, there were first-part flankers, which were always replaced by new second-part flankers after a variable time interval (SOA). A consequence of this procedure was that the first-part flankers were always completely irrelevant for the tasks, because participants never had to respond to them. The second-part flankers, though, were relevant on dual-task trials, as in the previous experiments.

Our main question was whether participants would still coprocess the flankers in dual tasks under these conditions. If, in the previous experiments, participants avoided the inhibition of flankers because these had to be used as stimuli for $\mathrm{T}_{2}$, they should now suppress at least the processing of the first-part flankers, because these would be irrelevant for $\mathrm{T}_{2}$. Accordingly, no FCE should be observed for these flankers. Whether the FCE should also be absent for the second-part flankers in dual tasks depends on whether participants start flanker processing after the selection of $\mathrm{R}_{1}$ or with cue encoding. If, for instance, the processing of the first-part flankers is suppressed by inhibition of their spatial location, then it seems likely that the inhibition will also extend to the second-part flankers. Participants may then start flanker processing in dual tasks only after the selection of $R_{1}$. Because there is also no need to process the flankers in single tasks, one would expect similarly small FCEs for both trial types in that case. However, if flanker processing is suppressed-for instance, by inhibition of individual flanker representations - then it could be possible that participants will start to process the second-part flankers after their onset. In this case, the FCEs should differ between single and dual tasks.

\section{Method}

Eight people ( 5 female, 3 male; mean age $=22$ years) participated in the experiment either for partial fulfillment of course requirements or for payment of $€ 5$ (U.S.\$6.50) per hour. All were right-handed (by self-report) and had normal or corrected-to-normal vision. The stimuli and procedure were similar to those in the previous experiment. Here, however, the flankers changed their identity with changed coloring. Thus, their congruency could also change. For example, congruent first-part flankers could be replaced by congruent second-part flankers, but they could also be replaced by incongruent or neutral ones. There were nine different congruency combinations of first-part and second-part flankers, which were equal in frequency and randomized across trials. Only three SOAs $(50,150$, or 350 $\mathrm{ms}$ ) were used. An SOA of $0 \mathrm{~ms}$ was not used because, in that case, flanker identity could not have changed within a trial. After a training block, 16 blocks of 96 trials were run in two separate 1-hr sessions.

\section{Results}

\section{Response Times}

$R T_{1}$

First-part flankers. First of all, to investigate the effect of the first-part flankers, we conducted a repeated measures ANOVA with congruency (congruent or incongruent), task mode (single task or dual task), and SOA $(50,150$, or $350 \mathrm{~ms})$ as factors. The analysis revealed no significant main effect of congruency, $F(1$, $7)=0.58, p=.470$. Overall mean response times were $626 \mathrm{~ms}$ for congruent and $629 \mathrm{~ms}$ for incongruent first-part flankers. However, there was a significant SOA $\times$ Congruency interaction, $F(2$, $14)=7.36, p<.01$, indicating a small congruency effect at an SOA of $150 \mathrm{~ms}$. The FCEs were $-3,22$, and $-8 \mathrm{~ms}$ for the SOAs of 50, 150, and $350 \mathrm{~ms}$, respectively.

Second-part flankers. An ANOVA with congruency (of the second-part flankers), SOA, and task mode as factors revealed a significant main effect of task mode, $F(1,7)=10.70, p<.05$. Latencies on dual-task trials were $52 \mathrm{~ms}$ longer than latencies on single-task trials (see Figure 6). Also, the main effect of congruency was reliable, $F(1,7)=39.20, p<.001$. The mean size of the FCE was $31 \mathrm{~ms}$. There was no significant Congruency $\times$ Task Mode interaction - that is, the FCE did not differ significantly between single $(34 \mathrm{~ms})$ and dual tasks $(30 \mathrm{~ms})$. The main effect of SOA was marginally significant $F(2,14)=3.49, p<.059$. On average, the latencies increased by $41 \mathrm{~ms}$ across SOAs.

Neutral. An analysis of the data for the neutral second-part flankers revealed a significant main effect of SOA, $F(2,14)=$ 21.20, $p<.001$. Latencies increased by $93 \mathrm{~ms}$ over the SOA range. The difference between the neutral trials for single and dual tasks failed to reach significance, $F(1,7)=5.15, p=.057$.

There was a significant difference between neutral and congruent flankers on dual-task trials, $F(1,7)=30.10, p<.001$, but not 

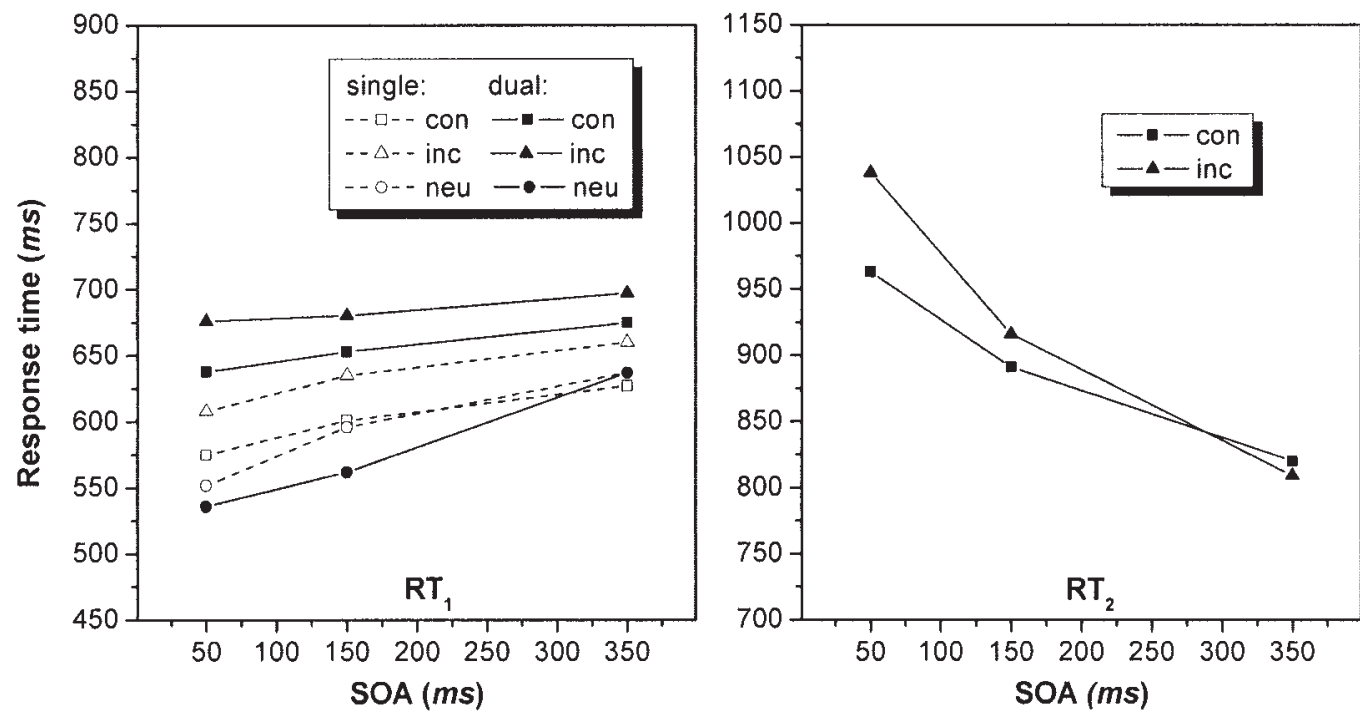

Figure 6. Response time as a function of stimulus onset asynchrony ( $\mathrm{SOA})$ for Task $1\left(\mathrm{RT}_{1}\right)$ and Task $2\left(\mathrm{RT}_{2}\right)$ in Experiment 4. single $=$ single task; dual $=$ dual task; con $=$ congruent; inc $=$ incongruent; neu $=$ neutral.

on single-task ones, $F(1,7)=1.19, p=.310$. Latencies on neutral trials were $77 \mathrm{~ms}$ shorter than those on congruent dual-tasks trials.

\section{$R T_{2}$}

The latencies of correct responses $\left(\mathrm{R}_{1}\right.$ and $\left.\mathrm{R}_{2}\right)$ were entered into an ANOVA with (second-part flanker) congruency (congruent or incongruent) and $\mathrm{SOA}(50,150$, or $350 \mathrm{~ms})$ as factors. This analysis revealed that the latencies decreased significantly with increasing SOA, $F(2,14)=81.80, p<.001$. The congruency effect of $31 \mathrm{~ms}$ was only marginally significant, $F(1,7)=3.84$, $p=.091$. However, there was significant Congruency $\times$ SOA interaction, $F(2,14)=8.33, p<.01$. The congruency effect decreased with increasing SOA (see Figure 6).

\section{Error Rates}

The mean error rate for $\mathrm{RT}_{1}$ was $3.83 \%$, and that for $\mathrm{R}_{2}$ (given that $\mathrm{R}_{1}$ had been correct) was $6.39 \%$. For $\mathrm{R}_{1}$, the error rates differed significantly between single $(4.34 \%)$ and dual tasks (3.33\%), $F(1,7)=9.81, p<.05$. Moreover, the error rates decreased with increasing SOA, $F(2,14)=6.52, p<.01$. Congruency of the first-part flankers had no significant effect on the error rates. Also, for neutral conditions, there were no significant effects. For $\mathrm{RT}_{2}$, there was a significant Congruency $\times \mathrm{SOA}$ interaction, $F(2,14)=5.20, p<.05$, indicating a decreasing effect with increasing SOA.

\section{Discussion}

The aim of this experiment was to examine whether participants are capable of suppressing flanker processing during $T_{1}$ in dual tasks. Here, we presented irrelevant first-part flankers for a variable time interval, which were then replaced by relevant secondpart flankers $\left(\mathrm{S}_{2}\right)$. Consequently, participants never had to respond to the first-part flankers, whereas a response to the second-part flankers was required only on dual-task trials. The color of the second-part flankers signaled whether a single task or dual tasks were required. As the results show, the first-part flankers produced no substantial FCE even when they were present for $350 \mathrm{~ms}$ (the only exception being a small effect at an SOA of $150 \mathrm{~ms}$, which is difficult to interpret). This indicates that the participants almost completely suppressed processing of the first-part flankers. In contrast, the second-part flankers were processed to some extent, as is implied by the corresponding FCEs (see Figure 6). However, these effects were rather small compared with those in the previous experiment, and they did not vary with SOA. Because the relevant flankers were not available before the cues arrived, it appears that the flankers were coprocessed only for a short time period after their appearance. Presumably, this happened because cue information had to be encoded anyway, and by this time, the replaced flankers were encoded to some extent, which produced some interference. This explains not only why the FCE was relatively small and constant across the SOAs but also why there was no significant FCE difference between single-task and dual-task trials. Altogether, the pattern of the FCEs suggests that under these conditions, the participants generally inhibited the spatial location of the flankers during $\mathrm{T}_{1}$.

This time, the SOA effects on $\mathrm{RT}_{1}$ were also similar for single and dual tasks. There was a small increase that was presumably completely attributable to waiting, as indicated by the data for the neutral conditions. However, $\mathrm{RT}_{1}$ was generally increased for dual tasks relative to single tasks. This suggests that when the cue signaled that a second task was required, participants increased their preparedness to some extent. In contrast to the previous experiment, though, the increase was not accompanied by an increased FCE. However, the earlier the preparedness was increased, the more time there was available for the target to produce some interference effect on $\mathrm{RT}_{2}$ (see Figure 6). The second response again showed the standard PRP effect. In any case, these results demonstrate that preparedness for a second task can be 
controlled independently, at least to some extent, from the degree of flanker coprocessing.

Altogether, the results demonstrate that participants are capable of suppressing flanker processing during $\mathrm{T}_{1}$ in dual tasks. In the present experiment, this was achieved by presentation of irrelevant first-part flankers. However, because this procedure is rather different from that of the standard flanker task, it is difficult to generalize our results. Therefore, in Experiment 5 we examined whether a serial processing strategy in dual tasks can also be induced in situations in which the flankers do not change their identity.

\section{EXPERIMENT 5}

Experiment 5 aimed to induce a serial processing strategy by increasing the percentage of single-task trials. Varying the relative percentages of trial types is a well-established means of manipulating processing strategies (e.g., Hommel, 1994; Logan \& Zbrodoff, 1979; Marble \& Proctor, 2000). Because the percentage of single-task trials in Experiment $3(62.5 \%)$ was obviously not sufficient to induce a serial strategy, here we administered blocks with $80 \%$ single-task trials and $20 \%$ dual-task trials. So as to have a direct comparison with a parallel strategy condition, we also included blocks with $80 \%$ dual-task trials and $20 \%$ single-task trials. We denote these block types as ST blocks and DT blocks, respectively. To ensure the specific percentages, we included no neutral trials.

Because both trial types could occur, participants would still have had to maintain a control structure that principally allowed single-task as well as dual-task processing. The extreme relative percentages of the trial types should nevertheless affect the general strategy of flanker coprocessing and also the relative preparation for one or the other type of trial. In view of our previous results, we can derive a number of predictions. For instance, the large percentage of dual tasks in DT blocks should lead to parallel processing of the target and flankers, which should be reflected by a substantial FCE in dual tasks. Moreover, the FCE should be of a similar size for the few single-task trials. Also, because participants should be prepared for dual tasks, $R_{2}$ should be relatively fast, whereas $\mathrm{RT}_{1}$ on single-task trials should be impaired relative to that in ST blocks. In contrast, in blocks with mainly single tasks, a more serial strategy should be applied, because the flankers will be irrelevant most of the time. This should produce a small FCE on single-task trials and also on the few dual-task trials. Also, because participants will be relatively unprepared for a second response, $\mathrm{RT}_{2}$ should be increased relative to that in DT blocks.

\section{Method}

12 individuals ( 9 female, 3 male; mean age $=22$ years) participated in the experiment either for partial fulfillment of course requirements or payment of $€ 5$ (U.S.\$6.50) per hour. All were right-handed (by self-report) and had normal or corrected-to-normal vision. The stimuli and procedure were similar to those in Experiment 3. Here, however, the relative percentage of single and dual tasks was varied between blocks. There were two block types: ST blocks (with 80\% single-task trials and 20\% dual-task trials) and DT blocks (with $80 \%$ dual-task trials and 20\% single-task trials). Neutral trials were not included. Single-task and dual-task trials were randomly mixed within each block, and color again indicated the trial type. The target and flankers always appeared simultaneously in white. After an
SCI of 50,150, or $350 \mathrm{~ms}$, the stimuli changed color. The coloring and meaning of the color match and mismatch were the same as in the corresponding previous experiments.

Altogether, 16 experimental blocks of 90 trials were run in two separate 1-hr sessions. The different block types were administered in runs of 4 blocks. That is, participants started with 4 blocks of one type and then performed 4 blocks of the other type. Before each block started, participants were informed about its type. In the second session, the same order was applied. Half of the participants started with ST blocks and then switched to DT blocks; for the other half, this order was reversed.

\section{Results}

\section{Response Times}

$R T_{1}$

The latencies of correct responses were entered into a repeated measures ANOVA with task mode (single task or dual task), congruency (congruent or incongruent), and block type (ST or DT) as factors. The factor SCI was excluded from the overall analysis because there were not enough trials for the trial type with the lower percentage in each block type. However, these data are shown in Figure 7. The effects of the SCI for the trial types with the higher percentage are reported below.

There was a significant main effect of block type, $F(1,11)=$ $19.70, p<.001$, indicating that response times were substantially longer in DT blocks (812 ms) than in ST blocks (662 ms). Furthermore, there was a significant main effect of task mode, $F(1$, $11)=6.62, p<.05$. However, there was also a significant Block Type $\times$ Task Mode interaction, $F(1,11)=24.60, p<.001$. The difference between single and dual tasks was much larger in ST blocks $(122 \mathrm{~ms})$ than in DT blocks $(10 \mathrm{~ms})$. There was also a significant main effect of congruency, $F(1,11)=62.10, p<.001$. And there was a significant Congruency $\times$ Block Type interaction, $F(1,11)=12.90, p<.01$. As expected, the FCE was larger in the DT blocks (76 ms) than in the ST blocks (34 ms). The data were also analyzed separately for the two task modes.

Single task. The analysis revealed a significant main effect of block type, $F(1,11)=33.80, p<.001$. On average, the participants were $106 \mathrm{~ms}$ slower on single-task trials when they occurred in DT blocks than when they occurred in ST blocks. Furthermore, there was a significant main effect of congruency (52 ms), $F(1$, $11)=37.30, p<.001$. However, there was also a marginally significant Block Type $\times$ Congruency interaction, $F(1,11)=$ $4.64, p=.054$. The FCE was smaller in ST blocks (33 ms) than in DT blocks ( $72 \mathrm{~ms}$ ). The latencies increased by $60 \mathrm{~ms}$ with increasing SCI for the single-task trials within ST blocks (see Figure 7), which was highly significant, $F(2,22)=12.90, p<.001$.

Dual task. For the dual-task trials, there was a significant main effect of block type, $F(1,11)=6.86, p<.05$. RT ${ }_{1}$ for dual-task trials was $94 \mathrm{~ms}$ shorter in ST blocks than in DT blocks. Furthermore, the effect of congruency was significant, $F(1,11)=19.50$, $p<.01$. However, there was also a significant Congruency $\times$ Block Type interaction, $F(1,11)=7.95, p<.05$. The FCE was 34 ms on dual-task trials in ST blocks, compared with $80 \mathrm{~ms}$ in DT blocks.

Finally, there was a significant main effect of SCI for the dual-task trials within DT blocks, $F(2,22)=3.66, p<.05$. Latencies increased by $99 \mathrm{~ms}$ with increasing SCI. 

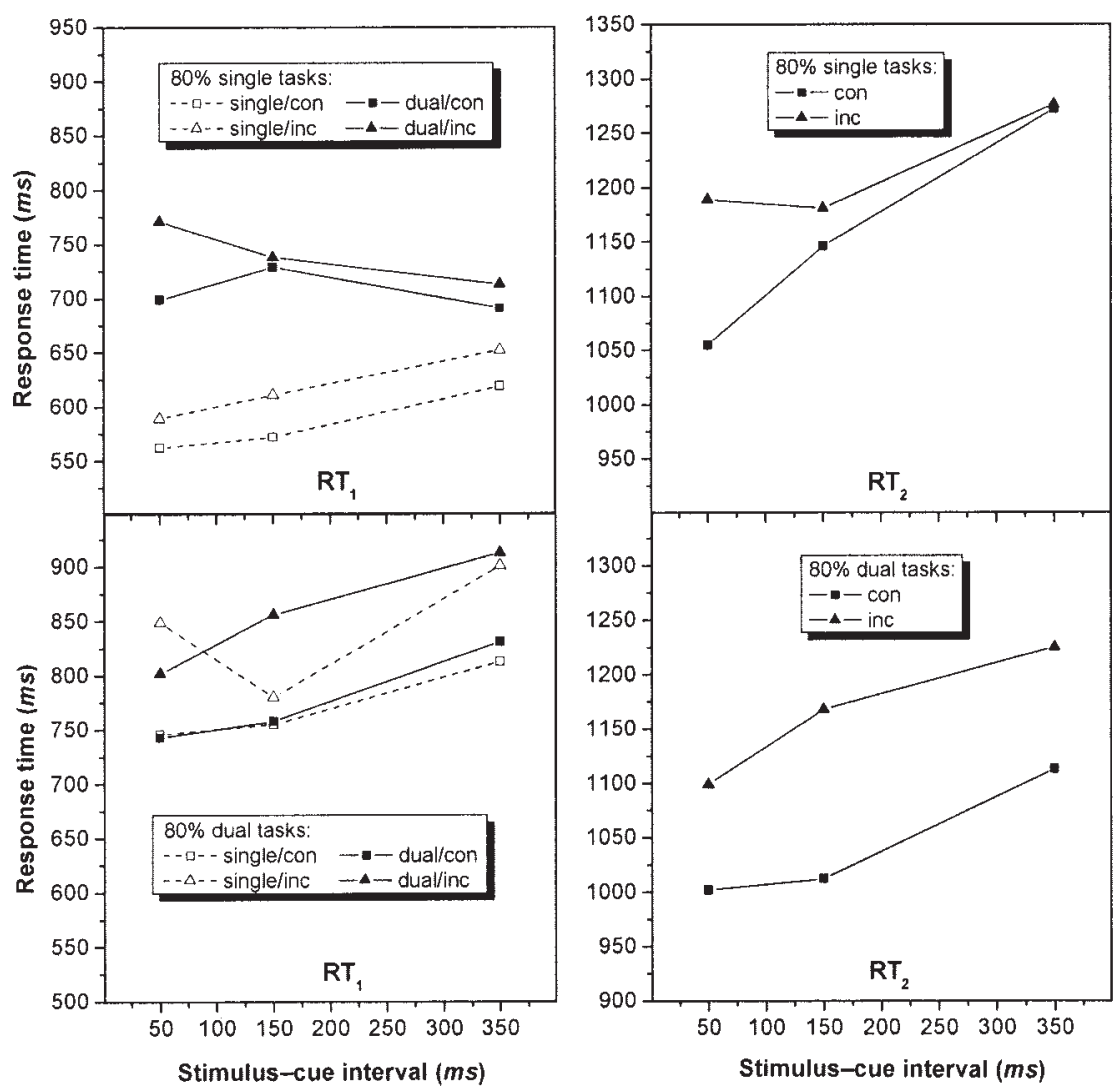

Figure 7. Response time as a function of stimulus-cue interval for Task $1\left(\mathrm{RT}_{1}\right)$ and Task $2\left(\mathrm{RT}_{2}\right)$ for $80 \%$ single-task and 20\% dual-task trials (upper panels) and $80 \%$ dual-task and 20\% single-task trials (lower panels 0 in Experiment 5. single $=$ single task; con $=$ congruent; dual $=$ dual task; inc $=$ incongruent .

$R T_{2}$

The latencies of correct responses $\left(R_{1}\right.$ and $\left.R_{2}\right)$ to the flankers were entered into a repeated measures ANOVA with block type (ST or DT) and congruency (incongruent or congruent) as factors. The analysis revealed a significant main effect of block type, $F(1$, $11)=11.90, p<.01$. Response times were $83 \mathrm{~ms}$ shorter in DT blocks than in ST blocks. Furthermore, there was a significant main effect of congruency (90 ms), $F(1,11)=18.20, p<.01$. However, there was also a significant Congruency $\times$ Block Type interaction, $F(1,11)=17.80, p<.01$. The congruency effect was larger in DT blocks (122 ms) than in ST blocks (57 ms).

Moreover, a separate ANOVA including SCI for the trials within DT blocks revealed a significant main effect of SCI, $F(2$, $22)=54.90, p<.001$. Mean response times were $1,051 \mathrm{~ms}, 1,090$ $\mathrm{ms}$, and $1,169 \mathrm{~ms}$ for the SCIs of 50,150 , and $350 \mathrm{~ms}$, respectively. Finally, there was a significant Congruency $\times$ SCI interaction, $F(2,22)=3.97, p<.05$. The congruency effect was 97 $\mathrm{ms}, 156 \mathrm{~ms}$, and $112 \mathrm{~ms}$ for the SCIs of 50, 150, and $350 \mathrm{~ms}$, respectively.

\section{Error Rates}

The mean error rate for $R_{1}$ was $4.39 \%$, and that for $R_{2}$ (given that $\mathrm{R}_{1}$ had been correct) was $4.47 \%$. The difference in error rate between single $(4.93 \%)$ and dual tasks for $\mathrm{R}_{1}(3.85 \%)$ was significant, $F(1,11)=6.52, p<.05$. Furthermore, there was a significant main effect of congruency, $F(1,11)=8.24, p<.05$ indicating that more errors occurred with incongruent (5.24\%) than with congruent flankers $(3.55 \%)$.

For $\mathrm{R}_{2}$, there was a significant main effect of block type, $F(1$, $11)=6.87, p<.05$. More errors occurred in ST blocks $(5.96 \%)$ than in DT blocks (2.97\%). However, there was a significant Block Type $\times$ Congruency interaction, $F(1,11)=5.95, p<.05$. There was a small congruency effect for DT blocks, whereas the effect was reversed for ST blocks. Moreover, there was a significant main effect of SCI, $F(2,22)=15.40, p<.001$. Error rate increased with increasing SCI.

\section{Discussion}

In Experiment 5, we investigated whether participants can be encouraged to use a serial processing strategy by increasing the percentage of single tasks. The procedure was similar to that of Experiment 3, except the percentage of single-task trials was increased to $80 \%$ in ST blocks. For comparison, there were also DT blocks with $80 \%$ dual-task trials. Thus, the specific question was whether a more serial strategy was applied in ST blocks than in DT blocks. As a number of effects reveal, this was indeed the case. 
First of all, the FCE in ST blocks was rather small (34 ms) only half the size of that in DT blocks (76 ms). Furthermore, $\mathrm{RT}_{1}$ was $150 \mathrm{~ms}$ shorter in ST blocks than in DT blocks. These effects show that in ST blocks, participants strongly suppressed flanker coprocessing and made little preparation for $\mathrm{T}_{2}$. As expected, the reduced preparation for dual tasks impaired performance for $T_{2}$. Presumably, in ST blocks, participants started to prepare for $\mathrm{T}_{2}$ only after the cue signaled a dual task. Obviously, this preparation consumed a large amount of resources and, consequently, increased $\mathrm{RT}_{1}$. However, the degree of flanker coprocessing was not increased, as indicated by the fact that FCEs did not differ between single and dual tasks.

In DT blocks, participants seemed to have generally been prepared for dual tasks. This had positive effects on $\mathrm{RT}_{2}$. However, it also impaired performance for $\mathrm{T}_{1}$, as reflected by the increased $\mathrm{RT}_{1}$ compared with that for single tasks in ST blocks. Also, the degree of flanker coprocessing was larger in DT blocks, as indicated by the increased FCE. It is interesting to note that if a single task was signaled in DT blocks, this had absolutely no effect on the selection of $R_{1}$. The flankers were coprocessed to the same degree as on dual-task trials, as indicated by the similar FCEs for the two trial types. Even the preparation for $\mathrm{T}_{2}$ was maintained during the processing for $\mathrm{T}_{1}$.

If we consider the data for the dominant trial types (i.e., single and dual tasks in ST and DT blocks, respectively), it is obvious that the FCE did not vary with SCI. This replicates the results of Experiment 3. The results again show that the degree of flanker coprocessing remains relatively constant during $\mathrm{T}_{1}$ on a trial.

Altogether, the data give a clear picture of the involved strategies adopted in the individual block types. They show that components of strategies, such as the degree of flanker coprocessing and preparation, are adapted in specific ways that depend on the context (see also Lien, Ruthruff, Remington, \& Johnston, 2005).

\section{GENERAL DISCUSSION}

The aim of the present experiments was to investigate the contribution of attentional strategies to the selection and processing of currently irrelevant stimuli. Although there have already been some studies showing that the degree to which irrelevant stimuli are processed depends on particular strategies (e.g., C. W. Eriksen \& St. James, 1986; Gratton et al., 1992; Logan et al., 1983), this question has been examined in a rather restricted way and mostly in single tasks. Here, we went a step further and investigated how different processing strategies affect the dualtask flanker paradigm. The main questions were whether flankers are coprocessed with the target to a larger degree when they have to be used as stimuli for a second task and, if so, for what reason. Accordingly, we compared performance in dual tasks with that in single tasks, and the FCE (i.e., the interference produced by the flankers on $\mathrm{RT}_{1}$ ) was taken as measure of the degree of flanker coprocessing during $\mathrm{T}_{1}$.

In our first experiment, single-task and dual-task trials were blocked. For single tasks, there was only a small FCE (and only at an SOA of $0 \mathrm{~ms}$ ), whereas a substantial FCE occurred on dual-task trials. This suggested that the participants processed the flankers differently, depending on the specific trial type. The question was whether this was because of deliberately chosen strategies or merely unavoidable task demands. For instance, it is conceivable that the more complex control structure in dual-task blocks increased the memory load, which, in turn, led to increased flanker coprocessing (cf. de Fockert et al., 2001; Lavie et al., 2004; Soto et al., 2005). To investigate this issue, in Experiment 2, we randomly mixed single-task and dual-task trials, and color information served as the cue to indicate whether a single task or dual tasks were required on a trial. Consequently, participants had to start each trial in the same state of preparedness (i.e., with the same general control structure), and only after the cue was presented was it possible to shift to a specific strategy for the trial. The data indicated that the flankers were again coprocessed to a larger degree in dual tasks than in single tasks. However, the difference in overall performance between single and dual tasks was reduced in comparison with that in Experiment 1. This suggests that the difference between the control structures for the two trial types was more extreme under the blocked conditions. Nevertheless, the fact that the FCEs still differed substantially between single and dual tasks, under randomized conditions, indicates that participants were capable of choosing specific processing strategies on the fly for the two trial types. This held across different trial-by-trial cuing procedures.

The FCE is an indication of parallel processing of the target and the flankers. Experiment 3 tested the hypothesis that participants chose such a parallel processing strategy because it would have been demanding to process the target and flankers serially (suppressing flanker processing during $\mathrm{T}_{1}$ and then processing flankers shortly thereafter for $\mathrm{T}_{2}$; see Tipper, 1985; Tipper \& Cranston, 1985; Tipper et al., 1994). Instead of processing inhibited stimuli, participants might have preferred to accept the higher performance costs (FCE) that stemmed from a parallel processing strategy. This could especially have been the case in our experiments with mixed trial types, in which the costs from parallel processing were substantial only for flankers that were incongruent and appeared relatively early.

On the basis of these considerations, in Experiment 3, we increased the costs for parallel processing by always presenting the flankers simultaneously with the target. Moreover, flanker onset and cue onset were separated. After a variable SCI, the color of both the target and the flankers changed, signaling the trial type. Under these conditions, a parallel strategy would lead to a substantial flanker coprocessing on single-task trials as well, at least until the cue appeared. This was exactly what we observed. The FCEs were considerably larger than those in Experiment 2, a result also attributable to the fact that the FCEs did not significantly decrease with increasing SCIs. Obviously, participants immediately started to coprocess the flankers when these appeared. Moreover, even on single-task trials, the coprocessing was not substantially reduced after the cue. These results indicate that participants still preferred parallel processing, despite the considerable costs.

Although FCEs were relatively large and remained almost constant across SCIs in Experiment 3, they again differed significantly between single-task and dual-task trials. However, because the participants did not know the trial type until the cue arrived, it is unlikely that this difference was a direct consequence of deliberately chosen strategies. Rather, it seems that participants improved their preparedness for $T_{2}$ after the cue signaled a dual task. This presumably increased the memory load, which, in turn, increased the interference (cf. de Fockert et al., 2001; Lavie et al., 2004). In any case, the procedure applied in Experiment 3 was not sufficient 
to encourage participants to apply a serial processing strategy in dual tasks.

These results raised the question of whether participants are capable of suppressing flanker coprocessing on dual-task trials. In Experiment 4, flankers were also presented simultaneously with the target, as in Experiment 3. However, after a certain interval (SOA), and together with cue onset (coloring), the flankers also changed their identity. Consequently, there were completely irrelevant first-part flankers (present until cue onset) and second-part flankers (present after cue onset). Under these conditions, first-part flankers should produce an FCE if a parallel processing strategy is still applied.

As the results of Experiment 4 show, except for a small effect at a single SOA, first-part flankers did not produce an FCE. Furthermore, although there was a significant FCE for the second-part flankers, it was rather small and, this time, did not differ between single and dual tasks. These results indicate that the participants applied a serial processing strategy and that they could indeed choose between serial and parallel processing on dual tasks.

However, because the applied procedure in Experiment 4 was rather different from that in the standard flanker task, especially in that flankers changed identity within a trial, it was difficult to generalize the results. Therefore, in Experiment 5, we applied a similar method as in Experiment 3 but induced different strategies by increasing or decreasing the percentage of single-task trials (cf. Logan \& Zbrodoff, 1979). In ST blocks, 80\% of the trials required single tasks, and $20 \%$ of the trials required dual tasks. The percentages were reversed in DT blocks. This manipulation strongly determined which processing strategy was applied. In ST blocks, participants adopted a serial processing strategy not only for the single tasks but also for the $20 \%$ dual-task trials. In contrast, in DT blocks, a parallel processing strategy was applied for dual tasks as well as for the $20 \%$ single tasks. Within both block types, the FCE did not differ between single-task and dual-task trials. Moreover, as in Experiment 3, it again did not change with SCI. Thus, under these conditions, participants adopted a general flanker coprocessing strategy in accordance with the dominant trial type and then also applied it for the other type. With respect to $T_{2}$, participants were less prepared in ST blocks than in DT blocks, which increased $\mathrm{RT}_{2}$ in the $\mathrm{ST}$ blocks.

\section{Implications for Spatial-Selection Models}

The results of the present study show that there is a strong tendency to coprocess currently irrelevant flankers to a larger degree in dual tasks than in single tasks. Because this was the case even when trial types were mixed, it can be concluded that the participants were generally capable of strategically adjusting the degree of flanker coprocessing on the fly during a trial. Moreover, the extent and flexibility with which the processing was adjusted goes beyond what has been previously proposed (for an exception, see Logan \& Gordon, 2001). In the dual-phase model (Gratton et al., 1992), for instance, it is assumed that strategies can merely determine whether a response is based on the output of the first, parallel phase - which leads to fast but unreliable responses-or on the output of the second, focused phase-which takes time but is more reliable. Our results, however, demonstrate that attentional selection is more than just deciding which information of two successive processing phases should be used for response selection.

Furthermore, because, according to the dual-phase model, the focused phase is essential for limiting the error rate, the relatively large degree of parallel processing throughout $T_{1}$ in some conditions of our experiments should have led to high error rates. This, however, was not the case. Rather, the error rates were similarly low in all of our experiments. This indicates that early spatial selection is not the only mechanism for controlling the degree to which stimulus information contributes to response selection. It is presumably the most easy and effective way of preventing irrelevant stimuli from affecting performance. However, if stimuli are irrelevant only for a short moment and then have to be processed, early spatial selection might be strenuous or costly. In this case, later mechanisms, such as those operating on objects, might be more appropriate for controlling the flow of information.

That participants are capable of suppressing flanker coprocessing during $T_{1}$ in dual tasks was shown in Experiment 4, in which the flankers changed identity within each trial, and also in the ST blocks of Experiment 5. Thus, it seems that participants suppress the processing of flankers whenever the flankers do not have to be used again for a second task on most trials.

The results of Experiment 5 also show that a dynamic trial-bytrial adjustment of the degree of flanker coprocessing is applied only when the percentages of single and dual tasks are almost balanced. If one task type dominates, a fixed strategy is applied. This suggests that the dynamic adjustment of flanker coprocessing also produces some costs, which are taken into account for the overall strategy in a block of trials.

Altogether, the results suggest that participants had a strong tendency to avoid serial processing of the target and flankers here because it would have been relatively demanding and costly for dual tasks. However, it is also possible that participants preferred a parallel strategy because they believed that the performance for $\mathrm{T}_{2}$ would benefit from the flanker coprocessing in $\mathrm{T}_{1}$. Although this hypothesis cannot definitively be excluded on the basis of the present data, we can examine whether such a belief would have been justified.

\section{Costs and Benefits of Flanker Coprocessing}

To see whether the coprocessing of flankers during $T_{1}$ was beneficial for $T_{2}$, we can compare its costs and benefits. Obviously, coprocessing produced considerable costs for $T_{1}$, especially for incongruent stimuli. To examine whether it also produced benefits that might have outweighed the costs, we considered $\mathrm{T}_{2}$ performance. In all of our experiments, when the SOA was varied, we observed the usual PRP effect. This did not change much across the different experiments. Thus, the PRP effect was rather unaffected by the different experimental manipulations, as was the absolute size of $\mathrm{RT}_{2}$.

For a further analysis, we took into account the FCE, which was assumed to reflect the degree of flanker coprocessing. If flanker coprocessing was beneficial for $\mathrm{T}_{2}$, then $\mathrm{RT}_{2}$ should have decreased with an increasing FCE. This, however, was obviously not the case. Rather, the coprocessing of incongruent flankers also produced costs on $\mathrm{RT}_{2}$. Moreover, even if we ignore these costs and merely consider $\mathrm{RT}_{2}$ for congruent flankers, there was no indication of any benefit. This can be seen in the lower panel of 
Figure 8, where $\mathrm{RT}_{2}$ for congruent stimuli is plotted against the FCE. For comparability, only those experiments in which the SOA was varied are shown. Obviously, there was no systematic relation between the FCE and $\mathrm{RT}_{2}$.

This analysis shows that $\mathrm{RT}_{1}$ increased with an increasing degree of coprocessing, whereas $\mathrm{RT}_{2}$ did not decrease but, rather, increased for incongruent flankers. Thus, if our participants thought that parallel processing in dual tasks would be beneficial for performance in $\mathrm{T}_{2}$, they were mistaken. This suggests that the supposed optimization of performance was not the sole reason for applying a parallel strategy in dual tasks. Rather, it seems that participants also took the mental costs for applying and adapting control structures into account (cf. Logan et al., 1983). A serial strategy would presumably have required a considerable control effort in dual tasks, in which responses would need to be made to previously suppressed flankers. The costs from this may have been less than the costs from parallel processing. Overall, it seems that participants chose a strategy that was a compromise between optimizing performance and minimizing overall control and processing effort.

\section{Costs and Benefits of Preparation}

A further aspect of the present study concerns the general preparation for the second task (cf. De Jong, 1995; Gottsdanker, 1980). In the experiments in which trial types were mixed, participants generally had to be prepared for dual tasks. Because this preparation substantially impaired performance for $T_{1}$, an interesting question is whether the degree of preparation improved per- formance for $\mathrm{T}_{2}$. If we consider $\mathrm{RT}_{1}$ (for congruent stimuli) as an indicator of the degree of preparation, we can examine whether there was any relation with $\mathrm{RT}_{2}$. Therefore, we plotted $\mathrm{RT}_{1}$ against $\mathrm{RT}_{2}$ for the congruent conditions in which the SOA was varied. As can be seen in the upper panel of Figure $8, \mathrm{RT}_{2}$ was independent of $\mathrm{RT}_{1}$. This suggests that increased preparation did not improve performance for $T_{2}$. This is also supported by the results of Experiment 5, in which preparation varied within a single experiment. These results show that the possibly increased preparation for $\mathrm{T}_{2}$ in DT blocks produced costs on $\mathrm{RT}_{1}$ of $94 \mathrm{~ms}$. However, the corresponding benefits on $\mathrm{RT}_{2}$ were only $83 \mathrm{~ms}$. Apparently, any preparation reflected by $\mathrm{RT}_{1}$ did not help much.

\section{Implications for Dual-Task Models}

An interesting question is whether dual-task models are compatible with our results. Because the PRP effects observed in the present study can presumably be explained by all dual-task models, we focus mainly on the effects on $\mathrm{RT}_{1}$. As mentioned in the introduction, the central bottleneck model (e.g., Pashler, 1984) cannot explain specific $\mathrm{S}_{2}$ effects on $\mathrm{RT}_{1}$, such as the FCE. One reason is that the model assumes that the perceptual stage of $T_{2}$ can proceed in parallel (and without capacity limit) with the stages of $\mathrm{T}_{1}$. These assumptions might be appropriate for dual tasks that are structurally very different. For explaining performance in dual-task flanker experiments, however, they are not sufficient and would have to be extended. One possibility could be to assume that there is also a direct path of response activation that bypasses the central bottleneck (Hommel, 1998). However, it is difficult to see how
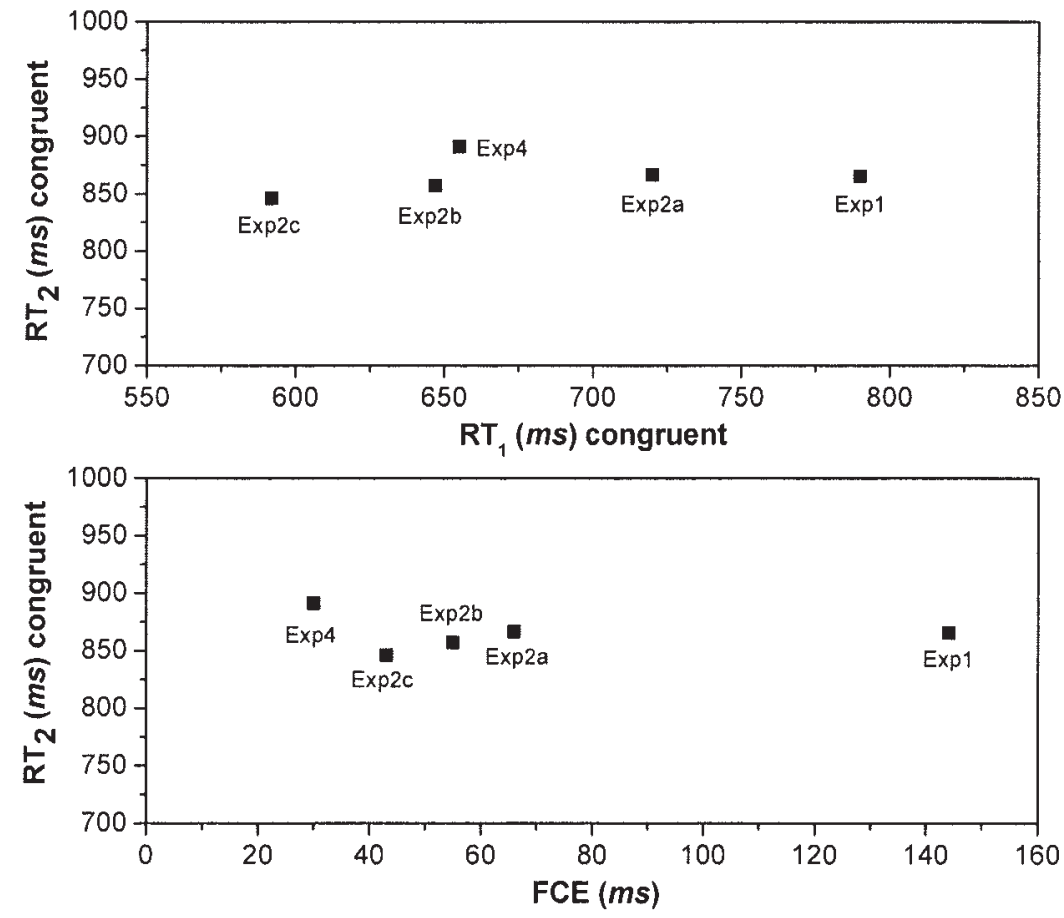

Figure 8. Response time for Task $2\left(\mathrm{RT}_{2}\right)$ for congruent stimuli as a function of response time for Task $1\left(\mathrm{RT}_{1}\right)$ across all experiments in which the stimulus onset asynchrony varied (upper panel) and as a function of the flanker congruency effect (FCE; lower panel; see text for details). 
such a mechanism could account for other aspects of our data, especially the influence of the different strategies.

The CCS model (cf. Tombu \& Jolicœur, 2003) would also have to be extended to account for the FCE. For instance, one could assume that a response conflict consumes some extra central capacity, which reduces performance for incongruent stimuli compared with congruent ones. This assumption would also explain the congruency effect on $\mathrm{RT}_{2}$. An interesting aspect of the CCS model with respect to the present data concerns the costs and benefits of parallel processing. As we have shown in our cost-benefit analysis, flanker coprocessing produced costs for $\mathrm{T}_{1}$ but did not facilitate $\mathrm{T}_{2}$. This is exactly what is predicted by the CCS model (cf. Tombu \& Jolicœur, 2003). Thus, if CCS is assumed, an optimal strategy for the present situations would have been to process target and flankers serially in dual tasks. It is interesting to note that Tombu and Jolicœur (2002) speculated that capacity sharing might be beneficial if both tasks in a dual-task situation are related. Our experiments clearly show that this is not the case.

The model that has least difficulty with explaining FCEs is Logan and Gordon's (2001) ECTVA, because it is a continuous flow model (C. W. Eriksen \& Schultz, 1979). Additionally, ECTVA includes an early selection mechanism for strategically controlling the degree of parallel processing. For instance, strict serial processing is accomplished by first allocating all spatial attention to the location of $S_{1}$ during $T_{1}$. For performance of $T_{2}$, attention is then redirected to $S_{2}$. Logan and Gordon assumed that this mechanism can, in principle, be used to realize all strategies from serial to parallel. However, ECTVA, like the dual-phase model (e.g., Gratton et al., 1992), predicts that a large degree of parallel processing will lead to a high error rate for incongruent flankers; consequently, the model holds that serial processing is usually the preferred mode of operation. Our results show that this is not the case. Rather, a serial selection strategy seems difficult to realize by early spatial mechanisms, and participants have a strong preference for parallel processing. Moreover, contrary to the prediction of ECTVA, the present data show that this does not necessarily lead to high error rates.

Thus, because its control mechanism is strongly based on early spatial selection, ECTVA faces problems similar to those of the dual-phase model for explaining the reliable performance that was achieved despite the relatively large degree of parallel processing in our experiments. Further research will have to show which other selection mechanisms have to be assumed to explain these phenomena.

\section{Conclusions}

Altogether, the results of this study demonstrate that the degree of flanker coprocessing in a dual-task flanker paradigm varies with processing strategy. Although participants are capable of applying a serial stimulus-processing strategy to targets and flankers in dual tasks, they have a strong tendency to coprocess the flankers to a large degree during $\mathrm{T}_{1}$, even if this impairs performance. This suggests that serial stimulus processing is rather demanding and requires much mental control. Under the present circumstances, participants applied a parallel processing strategy to targets and flankers to limit their mental-control effort.

\section{References}

Briand, K. A. (1994). Selective attention to global and local structure of objects: Alternative measures of nontarget processing. Perception \& Psychophysics, 55, 562-574.

Coles, M. G. H., Gratton, G., Bashore, T. R., Eriksen, C. W., \& Donchin, E. (1985). A psychophysiological investigation of the continuous flow model of human information processing. Journal of Experimental Psychology: Human Perception and Performance, 11, 529-553.

de Fockert, J. W., Rees, G., Frith, C. D., \& Lavie, N. (2001, March 2). The role of working memory in visual selective attention. Science, 291, 1803-1806.

De Jong, R. (1995). The role of preparation in overlapping-task performance. Quarterly Journal of Experimental Psychology: Human Experimental Psychology, 48(A), 2-25.

Desimone, R., \& Duncan, J. (1995). Neural mechanisms of selective visual attention. Annual Review of Neuroscience, 18, 193-222.

Eriksen, B. A., \& Eriksen, C. W. (1974). Effects of noise letters upon the identification of a target letter in a nonsearch task. Perception \& Psychophysics, 16, 143-149.

Eriksen, C. W., \& Hoffman, J. E. (1973). The extent of processing of noise elements during selective encoding from visual displays. Perception \& Psychophysics, 14, 155-160.

Eriksen, C. W., \& Schultz, D. W. (1979). Information processing in visual search: A continuous flow conception and experimental results. Perception \& Psychophysics, 25, 249-263.

Eriksen, C. W., \& St. James, J. D. (1986). Visual attention within and around the field of focal attention: A zoom lens model. Perception \& Psychophysics, 40, 225-240.

Flowers, J. H. (1990). Priming effects in perceptual classification. Perception \& Psychophysics, 47, 135-148.

Gottsdanker, R. (1980). The ubiquitous role of preparation. In G. E. Stelmach \& J. Requin (Eds.), Tutorials in motor behavior (pp. 355-371). Amsterdam: North-Holland.

Gratton, G., Coles, M. G. H., \& Donchin, E. (1992). Optimizing the use of information: Strategic control of activation of responses. Journal of Experimental Psychology: General, 121, 480-506.

Gratton, G., Coles, M. G. H., Sirevaag, E. J., Eriksen, C. W., \& Donchin, E. (1988). Pre- and poststimulus activation of response channels: A psychophysiological analysis. Journal of Experimental Psychology: Human Perception and Performance, 14, 331-344.

Hübner, R., \& Backer, G. (1999). Perceiving spatially inseparable objects: Evidence for feature-based object selection not mediated by location. Journal of Experimental Psychology: Human Perception and Performance, 25, 1556-1567.

Hübner, R., \& Volberg, G. (2005). The integration of object levels and their content: A theory of global/local processing and related hemispheric differences. Journal of Experimental Psychology: Human Perception and Performance, 31, 520-541.

Harms, L., \& Bundesen, C. (1983). Color segregation and selective attention in a nonsearch task. Perception \& Psychophysics, 33, 11-19.

Hommel, B. (1994). Effects of irrelevant spatial S-R compatibility depend on stimulus complexity. Psychological Research, 56, 179-184.

Hommel, B. (1998). Automatic stimulus-response translation in dual-task performance. Journal of Experimental Psychology: Human Perception and Performance, 24, 1368-1384.

Humphreys, G. W. (1981). Flexibility of attention between stimulus dimensions. Perception \& Psychophysics, 30, 291-302.

Kornblum, S., Hasbroucq, T., \& Osman, A. (1990). Dimensional overlap: Cognitive basis for stimulus-response compatibility-A model and taxonomy. Psychological Review, 97, 253-270.

Kramer, A. F., \& Jacobson, A. (1991). Perceptual organization and focused attention: The role of objects and proximity in visual processing. Perception \& Psychophysics, 50, 267-284.

Lavie, N., Hirst, A., de Fockert, J. W., \& Viding, E. (2004). Load theory 
of selective attention and cognitive control. Journal of Experimental Psychology: General, 133, 339-354.

Lien, M.-C., Ruthruff, E., Remington, R. W., \& Johnston, J. C. (2005). On the limits of advance preparation for a task switch: Do people prepare all the task some of the time or some of the task all the time? Journal of Experimental Psychology: Human Perception and Performance, 31, 299-315.

Lien, M.-C., Schweickert, R., \& Proctor, R. W. (2003). Task switching and response correspondence in the psychological refractory period paradigm. Journal of Experimental Psychology: Human Perception and Performance, 29, 692-712.

Logan, G. D., \& Gordon, R. D. (2001). Executive control of visual attention in dual-task situations. Psychological Review, 108, 393-434.

Logan, G. D., \& Schulkind, M. D. (2000). Parallel memory retrieval in dual-task situations: I. Semantic memory. Journal of Experimental Psychology: Human Perception and Performance, 26, 1072-1090.

Logan, G. D., \& Zbrodoff, N. J. (1979). When it helps to be misled: Facilitative effects of increasing the frequency of conflicting stimuli in an Stroop-like task. Memory \& Cognition, 3, 166-174.

Logan, G. D., \& Zbrodoff, N. J. (1982). Constraints on strategy construction in a speeded discrimination task. Journal of Experimental Psychology: Human Perception and Performance, 8, 502-520.

Logan, G. D., Zbrodoff, N. J., \& Fostey, A. R. W. (1983). Costs and benefits of strategy construction in a speeded discrimination task. Memory \& Cognition, 11, 485-493.

Logan, G. D., Zbrodoff, N. J., \& Williamson, J. (1984). Strategies in the color-word Stroop task. Bulletin of the Psychonomic Society, 22, 135138

Marble, J. G., \& Proctor, R. W. (2000). Mixing location-relevant and location-irrelevant choice-reaction tasks: Influences of location mapping on the Simon effect. Journal of Experimental Psychology: Human Perception and Performance, 26, 1515-1533.

McLeod, P. (1977). Parallel processing and the psychological refractory period. Acta Psychologica, 41, 381-396.

Meyer, D. E., \& Kieras, D. E. (1997). A computational theory of executive cognitive processes and multiple-task performance: Part 1. Basic mechanisms. Psychological Review, 104, 3-65.

Miller, J. (1991). The flanker compatibility effect as a function of visual angle, attentional focus, visual transients, and perceptual load: A search for boundary conditions. Perception \& Psychophysics, 49, 270-288.

Navon, D., \& Miller, J. (2002). Queuing or sharing? A critical evaluation of the single-bottleneck notion. Cognitive Psychology, 44, 193-251.

Paquet, L. (2001). Eliminating flanker effects and negative priming in the flankers task: Evidence for early selection. Psychonomic Bulletin \& Review, 8, 301-306.

Paquet, L., \& Craig, G. L. (1997). Evidence for selective target processing with a low perceptual load flankers task. Memory \& Cognition, 25, 182-189.

Paquet, L., \& Merikle, P. M. (1988). Global precedence in attended and nonattended objects. Journal of Experimental Psychology: Human Perception and Performance, 14, 89-100.

Pashler, H. (1984). Processing stages in overlapping tasks: Evidence for a central bottleneck. Journal of Experimental Psychology: Human Perception and Performance, 10, 358-377.

Pashler, H. (1990). Do response modality effects support multiprocessor models of divided attention? Journal of Experimental Psychology: $\mathrm{Hu}$ man Perception and Performance, 16, 826-842.

Pashler, H. (1991). Shifting visual attention and selecting motor responses: Distinct attentional mechanisms. Journal of Experimental Psychology: Human Perception and Performance, 17, 1023-1040.
Pashler, H. (1994). Dual-task interference in simple tasks: Data and theory. Psychological Bulletin, 116, 220-244.

Pashler, H., \& Johnston, J. C. (1989). Chronometric evidence for central postponement in temporally overlapping tasks. Quarterly Journal of Experimental Psychology: Human Experimental Psychology, 41(A), $19-45$.

Posner, M. I., \& Cohen, Y. (1984). Components of visual orienting. In H. Bouma \& D. G. Bouwhuis (Eds.), Attention and performance X: Control of language processes (pp. 531-556). London: Erlbaum.

Posner, M. I., Snyder, C. R. R., \& Davidson, B. J. (1980). Attention and the detection of signals. Journal of Experimental Psychology: General, 109, $160-174$.

Proctor, R. W., \& Vu, K.-P. L. (2002). Mixing location-irrelevant and location-relevant trials: Influence of stimulus mode on spatial compatibility effects. Memory \& Cognition, 30, 281-293.

Proctor, R. W., Vu, K.-P. L., \& Marble, J. G. (2003). Mixing locationrelevant and irrelevant tasks: Spatial compatibility effects eliminated by stimuli that share the same spatial codes. Visual Cognition, 10, 15-50.

Rogers, R. D., \& Monsell, S. (1995). Costs of a predictable switch between simple cognitive tasks. Journal of Experimental Psychology: General, 124, 207-231.

Smith, M. C. (1969). The effect of varying information on the psychological refractory period. Acta Psychologica, 30, 220-231.

Soto, D., Heinke, D., Humphreys, G. W., \& Blanco, M. J. (2005). Early, involuntary top-down guidance of attention from working memory. Journal of Experimental Psychology: Human Perception and Performance, 31, 248-261.

Steinhauser, M., \& Hübner, R. (in press). Automatic activation of taskrelevant representations in task-shifting. Memory \& Cognition.

Stroop, J. R. (1935). Studies of interference in serial verbal reactions. Journal of Experimental Psychology, 18, 643-662.

Telford, C. W. (1931). The refractory phase of voluntary and associative responses. Journal of Experimental Psychology, 14, 1-36.

Tipper, S. P. (1985). The negative priming effect: Inhibitory effects of ignored primes. Quarterly Journal of Experimental Psychology: Human Experimental Psychology, 37(A), 571-590.

Tipper, S. P., \& Cranston, M. (1985). Selective attention and priming: Inhibitory and facilitatory effects of ignored primes. Quarterly Journal of Experimental Psychology: Human Experimental Psychology, 37(A), 591-611.

Tipper, S. P., Weaver, B., Jerreat, L. M., \& Burak, A. L. (1994). Objectbased and environment-based inhibition of return of visual attention. Journal of Experimental Psychology: Human Perception and Performance, 20, 478-499.

Tombu, M., \& Jolicœur, P. (2002). All-or-none bottleneck versus capacity sharing accounts of the psychological refractory period phenomenon. Psychological Research, 66, 274-280.

Tombu, M., \& Jolicœur, P. (2003). A central capacity sharing model of dual-task performance. Journal of Experimental Psychology: Human Perception and Performance, 29, 3-18.

Vu, K.-P. L., \& Proctor, R. W. (2004). Mixing compatible and incompatible mappings: Elimination, reduction, and enhancement of spatial compatibility effects. Quarterly Journal of Experimental Psychology: Human Experimental Psychology, 57(A), 539-556.

Yantis, S., \& Johnston, J. C. (1990). On the locus of visual selection: Evidence from focused attention tasks. Journal of Experimental Psychology: Human Perception and Performance, 16, 135-149. 